\title{
Multiplatform Analysis of Primary and Metastatic Breast Tumors from the AURORA US Network identifies microenvironment and epigenetics drivers of metastasis
}

\section{Charles Perou ( $\nabla$ cperou@med.unc.edu )}

Lineberger Comprehensive Center, University of North Carolina, Chapel Hill, NC 27599.

https://orcid.org/0000-0001-9827-2247

\section{Susana Garcia-Recio}

University of North Carolina

Toshinori Hinoue4

Van Andel Institute

Gregory Wheeler

Nationwide Children's Hospital

Benjamin Kelly

Nationwide Children's Hospital

Ana Garrido-Castro

Dana-Farber Cancer Institute; Harvard Medical School

Tomás Pascual

SOLTI Breast Cancer Research Group

\section{Aguirre De Cubas}

Vanderbilt University Medical Center; Medical University of South Carolina

\section{Youli Xia}

University of North Carolina at Chapel Hill

\section{Marni McClure}

University of North Carolina; Johns Hopkins University

Andrei Rajkovic

Nationwide Children's Hospital

\section{Ezgi Karaesmen}

Nationwide Children's Hospital

\section{Markia Smith}

University of North Carolina

\section{Cheng Fan}

Lineberger Comprehensive Cancer Center

Chad Creighton 
Baylor College of Medicine https://orcid.org/0000-0002-6090-703X

Jay Bowen

Nationwide Children's Hospital https://orcid.org/0000-0001-6861-9043

Kristen Leraas

The Research Institute at Nationwide Children's Hospital

\section{Robyn Burns}

Translational Breast Cancer Research Consortium

\section{Sara Coppens}

Nationwide Children's Hospital

\section{Amy Wheless}

University of North Carolina

\section{Salma Rezk}

University of North Carolina

\section{Amy Garrett}

University of North Carolina

\section{Joel Parker}

University of North Carolina School of Medicine

\section{Kelly Foy}

Van Andel Institute

\section{Hui Shen}

van Andel

\section{Ben Park}

Vanderbilt University Medical Center

\section{lan Krop}

DFCl

\section{Carey Anders}

University of North Carolina https://orcid.org/0000-0001-9165-4074

\section{Julie Gastier-Foster}

Nationwide Children's Hospital

\section{Mothaffar Rimawi}

Baylor College of Medicine

\section{Rita Nanda}

University of Chicago https://orcid.org/0000-0001-5248-0876

\section{Nancy Lin}

Department of Medical Oncology, Dana-Farber Cancer Institute

\section{Claudine Isaacs}

Georgetown University

\section{P. Marcom}

Duke University Cancer Center 


\section{Anna Storniolo}

Indiana University School of Medicine

\section{Fergus Couch}

Mayo Clinic

\section{Uma Chandran}

UPMC Hillman Cancer Center; University of Pittsburgh

\section{Michael Davis}

UPMC Hillman Cancer Center; University of Pittsburgh

\section{Jonathan Silverstein}

University of Pittsburgh https://orcid.org/0000-0002-9252-6039

\section{Alexander Ropelewski}

Pittsburgh Supercomputing Center; Carnegie Mellon University

\section{Minetta Liu}

Mayo Clinic

\section{Susan Hilsenbeck}

Baylor College of Medicine

\section{Larry Norton}

Department of Medicine \& Office of the President, Memorial Sloan Kettering Cancer Center, New York, NY 10065, USA

\section{Andrea Richardson}

Department of Pathology, Brigham and Women's Hospital, Boston, MA 02115 https://orcid.org/00000001-5221-1094

\section{William Symmans}

University of Texas MD Anderson Cancer Center https://orcid.org/0000-0002-1526-184X

\section{Antonio Wolff}

Johns Hopkins University

\section{Nancy Davidson}

Fred Hutchinson Cancer Research Center and University of Washington

\section{Lisa Carey}

University of North Carolina https://orcid.org/0000-0003-2388-4649

\section{Adrian Lee}

University of Pittsburgh https://orcid.org/0000-0001-9917-514X

\section{Justin Balko}

Vanderbilt University Medical Center https://orcid.org/0000-0002-4263-5974

\section{Katherine Hoadley}

University of North Carolina at Chapel Hill https://orcid.org/0000-0002-1216-477X

\section{Peter W. Laird}

Van Andel Institute https://orcid.org/0000-0001-9117-3641

\section{Elaine Mardis}


The Ohio State University College of Medicine https://orcid.org/0000-0002-5892-1553

\section{Tari King}

Dana-Farber Cancer Institute; Harvard Medical School

\section{Biological Sciences - Article}

\section{Keywords:}

Posted Date: January 11th, 2022

DOl: https://doi.org/10.21203/rs.3.rs-1221704/v1

License: (c) (i) This work is licensed under a Creative Commons Attribution 4.0 International License. Read Full License 
Multiplatform Analysis of Primary and Metastatic Breast Tumors from the AURORA US Network identifies microenvironment and epigenetics differences as drivers of metastasis

Susana Garcia-Recio ${ }^{1, *}$,Toshinori Hinoue ${ }^{2, *}$, Gregory L Wheeler ${ }^{3} *$, Benjamin J. Kelly ${ }^{3} *$, Ana C. Garrido-Castro ${ }^{4, *}$, Tomas Pascual ${ }^{1,6}$, Aguirre A. De Cubas ${ }^{7,8}$, Youli Xiaa ${ }^{1,9}$, Marni B. McClure $^{1,10}$, Andrei Rajkovic ${ }^{3}$, Ezgi Karaesmen ${ }^{3}$, Markia A. Smith ${ }^{1}$, Cheng Fan ${ }^{1}$, Chad J. Creighton $^{11}$, Jay Bowen ${ }^{3}$, Kristen Leraas ${ }^{3}$, Robyn T. Burns ${ }^{12}$, Sara Coppens ${ }^{3}$, Amy Wheless ${ }^{1}$, Salma Rezk ${ }^{1}$, Amy L. Garrett ${ }^{1}$, Joel S. Parker ${ }^{1}$, Kelly K. Foy ${ }^{2}$, Hui Shen ${ }^{2}$, Ben H. Park ${ }^{7}$, Ian Krop $^{4}$, Carey Anders ${ }^{13}$, Julie Gastier-Foster ${ }^{3}$, Mothaffar F. Rimawi ${ }^{11}$, Rita Nanda ${ }^{14}$, Nancy U. Lin $^{4}$, Claudine Isaacs ${ }^{15}$, P. Kelly Marcom ${ }^{13}$, Anna Maria Storniolo ${ }^{16}$, Fergus J. Couch ${ }^{17}$, Uma Chandran $^{18}$, Michael Davis ${ }^{18}$, Jonathan Silverstein ${ }^{18}$, Alexander Ropelewski ${ }^{19}$, Minetta C. Liu ${ }^{17}$, Susan G. Hilsenbeck ${ }^{11}$, Larry Norton ${ }^{20}$, Andrea L. Richardson ${ }^{10}$, W. Fraser Symmans ${ }^{21}$, Antonio C. Wolff ${ }^{10}$, Nancy E. Davidson ${ }^{22}$, Lisa A. Carey ${ }^{1}$, Adrian V. Lee ${ }^{18, \#}$, Justin M. Balko ${ }^{7, \#,}$ Katherine A. Hoadley ${ }^{1, \#}$, Peter W. Laird ${ }^{2, \#}$, Elaine R. Mardis ${ }^{3, \#}$, Tari A. King, ${ }^{4,5, \#}$, Charles M. Perou $^{1, \#, A}$ on behalf of the AURORA US Network.

1. University of North Carolina, Chapel Hill, NC

2. Van Andel Institute, Grand Rapids, MI

3. Nationwide Children's Hospital, Columbus, $\mathrm{OH}$

4. Dana-Farber Cancer Institute, Harvard Medical School, Boston, MA

5. Division of Breast Surgery, Brigham and Women's Hospital.

6. SOLTI Cancer Research Group, Barcelona, Spain

7. Vanderbilt University Medical Center, Nashville, TN

8. Medical University of South Carolina, Charleston, SC

9. Cellarity, Cambridge, MA

10. Johns Hopkins University, Baltimore, MD

11. Baylor College of Medicine, Houston, TX

12. Translational Breast Cancer Research Consortium

13. Duke University, Durham, NC

14. University of Chicago, Chicago, IL

15. Georgetown University, Washington DC

16. Indiana University School of Medicine, Indianapolis, IN

17. Mayo Clinic, Rochester, MN

18. UPMC Hillman Cancer Center, University of Pittsburgh, Pittsburgh, PA

19. Pittsburgh Supercomputing Center, Carnegie Mellon University, Pittsburgh, PA

20. Memorial Sloan Kettering Cancer Center, New York, NY

21. MD Anderson Cancer Center, Houston, TX

22. Fred Hutchinson Cancer Research Center, University of Washington, Seattle, WA

* Equal contribution first authors

\# Equal Contribution Senior authors

${ }^{\mathrm{A}}$ Correspondence should be addressed to

Charles M. Perou

Lineberger Comprehensive Cancer Center

Marsico Hall, 5th floor, CB\#7599

125 Mason Farm Road

The University of North Carolina at Chapel Hill 
Chapel Hill, NC, 27599

cperou@med.unc.edu

phone: 919-843-5740

1

3

\begin{abstract}
Patients with metastatic breast cancer (MBC) typically have short survival times and their successful treatment represents one of most challenging aspects of patient care. This poor prognostic behavior is in part due to molecular features including increased tumor cell clonal heterogeneity, multiple drug resistance mechanisms, and alterations of the tumor microenvironment. The AURORA US Metastasis Project was established with the goal to identify molecular features specifically associated with metastasis. We therefore collected and molecularly characterized specimens from 55 metastatic breast cancer (BC) patients representing 51 primary cancers and 102 metastases. The 153 unique tumors were assayed using RNAseq, tumor/germline DNA exomes and low pass whole genome sequencing, and global DNA methylation microarrays. We found intrinsic molecular subtype differences between primary tumors and their matched metastases to be rare in triple negative breast cancer (TNBC)/Basallike subtype tumors. Conversely, tumor subtype changes were relatively frequent in estrogen receptor positive (ER+) cancers where $30 \%$ of Luminal A cases switched to Luminal B or HER2-enriched (HER2E) subtypes. Clonal evolution studies identified changes in expression subtype coincident with DNA clonality shifts, especially involving HER2 amplification and/or the HER2E expression subtype. We further found evidence for ER-mediated downregulation of genes involved in cell-cell adhesion in metastases. Microenvironment differences varied according to tumor subtype where ER+/Luminal metastases had lower fibroblast and endothelial cell content, while TNBC/Basal-like metastases showed a dramatic decrease in B cells and T cells. In $17 \%$ of metastatic tumors, we identified DNA hypermethylation and/or focal DNA deletions near HLA-A that were associated with its' significantly reduced expression, and with lower immune cell infiltrates. We also identified low immune cell features in brain and liver metastases when compared to other metastatic sites, even within the same patient. These findings have direct implications for the treatment of metastatic breast cancer patients with immune- and HER2-targeting therapies and suggest potential novel therapeutic avenues for the improvement of outcomes for some patients with MBC.
\end{abstract}




\section{Introduction}

A great deal of effort has gone into understanding the molecular causes of Metastatic Breast Cancer (MBC), to which 45,000 individuals/year succumb in the United States ${ }^{1}$. An early focus on metastatic disease has been to identify somatic alterations that might be unique to this setting, and/or that may be clinically actionable, especially when metastasis surgical resection may not be a viable option. Numerous seminal publications on MBC genomics have shown that almost no recurrent mutations are unique to the metastatic landscape, with perhaps the exception of ESR1 mutations, most of which are a thought to be tied to endocrine therapy resistance ${ }^{2,3}$. Instead, modestly increased frequencies of known pathogenic somatic variants (i.e., TP53, $P T E N, R B 1$ ) have been identified in metastases, as have similarly modest increases in the frequency of DNA amplifications/deletions ${ }^{2,4}$. Thus, much of the aggressive behavior of metastatic disease remains unexplained by DNA-based changes, invoking a multi-omic evaluation of this disease setting. Among the most impactful therapeutic advances in MBC has been the development and use of CDK4/6 inhibitors ${ }^{5-7}$, novel HER2-directed agents ${ }^{8,9}$, and immune checkpoint inhibitors (ICI) targeting CTLA4, PD-1 or PD-L1 ${ }^{10-12}$. These latter therapies target the immunosuppressive tumor immune microenvironment, thus highlighting the importance of non-tumor intrinsic factors as a major determinant of patient outcomes. In BC, ICIs have gained a role in both the early stage and metastatic settings, albeit with some mixed results in the clinical trial setting ${ }^{13}$, thus highlighting the need for an improved understanding of the MBC intrinsic and extrinsic landscapes, especially with an eye towards tumor-immune interactions.

\section{Results}

\section{Clinical features of the cohort and global genomic patterns}

A consortium of academic medical centers in the USA was formed (AURORA Metastatic Project) based upon the infrastructure of the Translational Breast Cancer Research Consortium (TBCRC), in order to pursue a multi-platform genomic study of matched metastatic and primary BCs, similar to The Cancer Genome Atlas (TCGA) effort on primary BCs ${ }^{14}$. Eligibility criteria for this retrospective study included the availability of a fresh frozen metastatic specimen, its associated primary tumor (fresh frozen or formalin-fixed paraffin embedded (FFPE)), a source of normal DNA, and corresponding tumor pathology and molecular analyte metrics (Fig. 1a). These requirements identified 55 patients, including 19 cases with more than one metastasis analyzed; 
20 patient samples were collected at autopsy (representing the patients with more than one metastasis). The clinical demographics of this group constituted a young cohort with a median age at primary diagnosis of 49 years, of which $18 \%$ were African American and $7 \%$ of Hispanic ethnicity. The distribution of clinical subtypes showed an increased frequency of patients with TNBC (34\%), relative to early-stage disease. In addition, 30\% of patients were ER+/HER2-, $11 \%$ were ER+/HER2+ and $7 \%$ were ER-/HER2+. In the metastatic setting, these patients received a median of three lines of systemic therapy. As might be expected, the overall survival of these patients was generally poor, and differed according to clinical subtype (Extended Data Fig.1). Metastases were obtained from multiple sites with the most common being liver $(n=28)$, lung ( $n=13)$, lymph nodes $(n=12)$, brain $(n=11)$, and 16 other sites; the relationship between clinical or genomic subtype, and site of metastasis, are shown in Extended Data Fig.2. Additional clinical demographics are shown in Supplementary Table 1.

Tumor DNA and RNA were isolated from each specimen and utilized, assuming sufficient quality and quantity, in four different genomic assays: DNA exomes and low-pass whole genome sequencing (tumor and normal), whole transcriptome RNAseq using rRNA depletion, and DNA methylation microarrays. In total, 88/153 specimens had all 4 assays successfully performed and 141/153 had 3 of 4 completed (Fig. 1b); this multi-platform genomic data set of 102 metastases and 51 paired primary tumors thus represents an unprecedented resource for the study of MBC. Global profiling of the DNA methylation landscape using the top 5000 most variably methylated CpGs displaying cancer-associated hypermethylation showed a remarkable conservation of overall methylation profiles within most of the primary tumor-metastasis pairs (Fig. 1c); indeed, $32 / 36$ tumor-metastasis pairs assayed showed the highest correlation to each other. Similar to the DNA methylation findings, gene expression-based hierarchical clustering using a 1710 gene breast tumor "intrinsic" list ${ }^{15}$ also identified the individuality of each tumor-metastasis pair, where $31 / 39$ pairs were co-clustered in the dendrogram (Fig. 1d), as seen in other studies of breast tumor metastases ${ }^{16,17}$. Lastly, the somatic mutation landscape identified TP53, KMT2C and PIK3CA as the most frequently mutated genes, together with the presence of ESRI mutations in metastases from ER+ patients (Fig. 1e). Similarly, the majority of somatic mutations within bona fide BC driver genes identified in TCGA that were found in AURORA primary tumors, were also present in the paired metastasis (Fig. 1e). The clinical and genomic data of AURORA- 
US Metastatic Project are available on a unique installation of cBioPortal (https://cbioportal.dbmi.pitt.edu/), and in dbGAP and GEO.

\section{Gene expression subtype switching and genomic signature differences}

As a first step to evaluate gene expression differences between primary tumors and their metastases, we performed PAM50 molecular subtyping from RNAseq data for each of the 123 specimens ${ }^{15,18}$, and evaluated molecular subtype concordance within each patient (Fig. 2a, b). Of the 39 RNAseq cases tested (39 patients with a primary tumor and one or more metastasis), 13/39 showed subtype "switching" between the primary and its associated metastasis. We do note that the normal-like distinction typically reflects low tumor cellularity, therefore, if we disregard switching to or from the normal-like group, then the basal-like phenotype is the most stable with $15 / 16$ pairs being basal-like in all specimens. Conversely, the "luminal" phenotypes that include Luminal A (LumA), Luminal B (LumB) and HER2-Enriched (HER2E), experienced subtype switching in $8 / 19$ patients.

To determine if any systematic differences exist between primary and metastatic tumors, we performed supervised learning analyses specifically comparing primary tumors to various groupings of the metastases. We first transformed the gene expression data into a set of 749 previously published gene expression signatures representing many features of tumor cells and their microenvironment including $>100$ signatures of immune cells, which showed significant correlation with pathologist assessed percent immune cell infiltration and with DNA methylation-based assessments of leukocyte infiltration ${ }^{19,20}$ (Extended Data Fig.3a-d). The complete list of signatures used is shown in Supplementary Table 2 and includes 48 signatures from the Molecular Signature Database ${ }^{21}$ and 701 signatures from 128 publications largely summarized in Garcia-Recio et al. ${ }^{16}$. Throughout our analyses, we relied on multiple validated immune cell signatures, including many that have shown prognostic and predictive information $^{18,20,22,23}$, as our main measures of immune cell presence/involvement. These immune signatures are focused on adaptive immunity and include CIBERSORT signatures of $\mathrm{T}$ cells and $\mathrm{B}$ cells ${ }^{24}$, and signatures of cooperating immune cells including an IgG signature ${ }^{25}$, a B cell/T cell cooperativity signature ${ }^{26}$ and a GP2-Immune-Metagene signature (see Methods). We performed supervised analyses of all primary tumors versus all metastases using this library of signatures and identified 135 signatures as being differentially expressed (q-value <0.05) 
125 (Extended Data Fig.4a) including signatures of fibroblasts/stromal cells, endothelial cells, and many adaptive immunity signatures as being lower in metastasis. However, when supervised analyses are performed within a gene expression subtype, which is known to associate with the likelihood of metastasis to a given site ${ }^{27,28}$, then subtype-specific differences are observed (Fig. 2c, d). Specifically, Luminal/ER+ subtype metastasis (LumA, LumB, and HER2E combined) showed low expression of fibroblast and endothelial signatures in metastases, and very few adaptive immune features were different. Conversely, Basal-like/TNBC metastasis, had significantly lower expression of many adaptive immune features including multiple T cells, B cells, NK cells and antigen presentation, while signatures of fibroblasts and endothelial cells were unchanged (Fig. 2d). Therefore, analyses of all tumors separated as primaries or metastases masked the subtype-specific microenvironment results.

We next asked if there were expression signature differences according to site of metastasis, and for these analyses we focused on the 3 most frequent sites of distant metastasis (i.e., liver, lung, and brain). Supervised learning of primary tumors versus their paired brain metastases yielded 48 signatures as being lower in brain metastasis, most of which were features of immunity and fibroblasts/stromal cells (Extended Data Fig.4b). Supervised analysis of liver metastases versus their primary tumors yielded 22 signatures as differentially expressed (Extended Data Fig.4c), while a similar analysis of lung metastases yielded no significant signatures. The small number of differentially expressed features suggested that we may be limited by our sample size, therefore, we obtained a second data set of primary tumor-metastasis pairs from our UNC Rapid Autopsy Program (RAP cohort that included 2 primary-metastasis pairs, 15 primary-multiple metastatic pairs, and 6 unique primaries and 1 metastasis, represented by 101 specimens with RNAseq data), and a third data set from the public domain that had 102 primary tumormetastasis pairs from the GEICAM/2009-03 ConvertHER trial (GEICAM cohort, 102 primary and 102 metastatic tumors, RNAseq only) ${ }^{16}$. Using this combined cohort of paired primary tumor-liver metastasis data ( $\mathrm{n}=61$ tumors, 28 primary and 33 metastases) yielded a larger set of significant signatures that now included many adaptive immunity signatures as being lower in liver metastasis (Extended Data Fig.4e). In addition, the combined cohort allowed us to refine our analysis of brain metastases in the setting of basal-like/TNBC phenotype ( $\mathrm{n}=16$ tumors, 5 primary and 11 metastasis), which also yielded more significant signatures including upregulated cell differentiation-related signatures and lower immune and stromal-related signatures 
(Extended Data Fig.4d). Lastly, the combined analysis of primary tumor-lung metastases ( $n=40$ tumors, 18 primary and 22 metastases) still yielded no significant signatures.

These comparative analyses suggest that immune features may systematically vary according to site of metastasis. To directly address this hypothesis, we performed 2 additional analyses. First, we took advantage of the combined AURORA-RAP data sets that contain 15 patients with at least 2 metastases analyzed by RNAseq, one of which is from the liver, to examine immune signature levels in different metastatic sites within the same patient. This analysis showed that in 9/15 patients the lowest immune cell signature levels of the GP2-Immune-Metagene signature were in liver metastases (Extended Data Fig.5a, b), and that in many of these patients, the immune signature is reduced in the liver metastases relative to the matched primary but is either unchanged or increased in the lung metastases (Extended Data Fig.5a, b). Second, we directly performed supervised learning using the combined AURORA-RAP-GEICAM cohort, comparing liver to lung metastases, and liver to lymph node metastases, both of which demonstrated significantly decreased immune signatures in liver metastases (Supplementary Table 3). We also compared liver metastases vs. brain metastases and saw 100 differential signatures that were primarily non-immune-related (with the exception of higher $\gamma \delta \mathrm{T}$ cells in brain metastases). When brain metastases are compared to lung or to lymph node metastases, brain also demonstrated decreased expression of immune-associated signatures. In summary, our site of metastasis comparative analyses revealed that both liver and brain, on average, have decreased immune cell signature levels regardless of the primary tumor phenotype.

\section{$H L A-A$ dysregulation and impact on anti-tumor immunity}

The decreased expression of an HLA metagene signature in basal-like/TNBC metastases led us to closely examine the multi-platform data of the individual genes comprising this signature, including $H L A-A,-B,-C$ and $B 2 M$. Examining promoter $C p G$ islands for $H L A-A,-B,-C$, and $B 2 M$, we identified $H L A-A$ methylation in 23 tumors (12 patients), and in which $H L A-A$ promoter methylation was more frequent in metastases (Fig. 3a, black bars) than primary tumors. In contrast, only 3 tumors ( 1 patient) demonstrated methylation at $H L A-B$ and only 1 tumor had $H L A-C$ or $B 2 M$ methylated (Fig. 3a), all of which displayed $H L A-A$ methylation. DNA copy number analysis also demonstrated 23 samples from 8 patients with significant focal deletions in this region $(\log 2$ ratio $<-0.75$, and focal mean difference $>0.25)$, but in only 14 samples from 3 
patients were these focal deletions near to an $H L A$ gene ( $<40 \mathrm{~kb}$ ) (Fig. 3b). From these 14 samples only 3 tumors ( 2 patients) had RNAseq data, and these focal deletions appeared nominally mutually exclusive from samples with $H L A-A$ methylation (Fig. 3c). Other HLA class I-associated DNA methylation events appeared to be rare, except for TAPBP. Consistent with a functional role of these events, metastatic samples with $H L A-A$ methylation or focal deletion had reduced mRNA of MHC class I genes and multiple immune signatures compared to their matched primaries (Fig. 3c and Extended Data Fig.6a, b). Supporting a role for a lack of immune-editing required in instances where MHC-I gene expression was downregulated in the metastasis, these $H L A-A,-B,-C$ altered samples also demonstrated a higher degree of $H L A-A$ predicted neoantigens (Fig. 3d). We also analyzed the relationship between HLA-A mRNA expression in primary tumors with paired metastases relative to immune signatures in the RAP data set of 17 primary tumor-metastasis pairs (17 primaries and 76 metastases) and identified the same relationship of low HLA-A mRNA and low/lower immune cell gene expression features, which again was the most frequent in basal-like/TNBC metastatic cancers (Extended Data Fig.6b, c).

Interestingly, we noted a strong inverse association of $H L A-A$ predicted neoantigens with $H L A-A$ gene expression, as opposed to $H L A-B$ or $-C$, in basal-like samples from both primaries and metastases (Extended Data Fig.7a). In basal-like primary and metastatic tumors, those tumors with HLA-A genetic or epigenetic alterations had significantly higher numbers of MHC-Iassociated neoantigens, which was not driven by a higher tumor mutational burden (TMB), further suggesting a lack of immune editing (Fig. 3d); particularly, patient AER2 showed more than 50 times higher neoantigen load in primary and liver metastasis compared to other patients. In this patient, $H L A-A$ was methylated in the AER2 primary and metastatic tumors and $H L A-A$ mRNA and immune signatures were even lower in the AER2 liver metastasis. In contrast, nonbasal-like primary and metastatic tumors demonstrated higher TMB and MHC-I neoantigens in cases with MHC-I genetic or epigenetic alterations than all others, suggesting that the increased neoantigen burden in these tumors was primarily a function of increased mutation load rather than the lack of immune editing (Fig. 3e). Moreover, a general decrease in $H L A-A,-B,-C$, as well as $B 2 M$ gene expression, was observed in basal-like samples with $H L A-A$ genetic or epigenetic alterations, likely reflecting a broader decrease in effector cell-mediated interferonregulated gene transcription resulting from $H L A-A$ deletion or hypermethylation (Fig. 3f). Taken 
together, these results point toward a high selective pressure on MHC-I-restricted neoantigens and CD8 T cell-mediated immunity, as well as MHC-I gene expression in basal-like BC. Of note, more pronounced downregulation of MHC-I genes was observed in metastatic samples procured after increased lines of metastatic therapy (Fig. 3g), regardless of subtype, supporting a paradigm where sequential chemotherapeutic regimens may select for lower antigen presentation in general.

Many cases with MHC-I genetic or epigenetic alterations, and thus MHC-I gene expression downregulation in the metastatic sample, tended to be of the basal-like subtype. To explore this more directly, we tested the association of primary/metastasis-specific downregulation of an MHC-I metagene signature composed of a composite expression of HLA-A, HLA-B, HLA-C, $B 2 M, T A P 1, T A P 2, N L R C 5$, between metastasis and matched primary, according to intrinsic subtype. Across the AURORA and RAP datasets, only basal-like BCs demonstrated consistent and significant downregulation of the MHC-I metagene signature in metastatic disease (Fig. 3h). This downregulation was observed for each of $H L A-A,-B$, and $-C$ genes only in basal-like tumors (Extended Data Fig.7b, c). Changes in gene expression for $H L A-A,-B$, and $-C$ genes were consistently altered within a given metastatic sample, supporting common regulation of all three genes, rather than gene-specific regulation (Extended Data Fig.7b, c).

To determine how antigen presentation via MHC-I expression and associated neoantigens may impact or reshape the tumor-immune microenvironment, we performed CIBERSORTx ${ }^{29}$ deconvolution on RNAseq data in 'relative mode' (i.e. all immune cell fractions are represented as a percent of total immune infiltrate). We constructed a correlation matrix that was further analyzed by unsupervised hierarchical clustering. We observed four associated clusters of features, two of which reflected positive feature correlation and two negative feature correlation patterns (Fig. 3i). The first positive cluster reflected associations of MHC-I neoantigens, specifically those with predicted binding affinity to $H L A-A$ and $-\mathrm{C}$, with tumor-associated $\mathrm{M} \varnothing$ macrophages, regulatory T (Treg) cells, and $\gamma \delta \mathrm{T}$ cells. The second positive cluster showed enrichment of cytotoxic CD8 T cells, memory activated CD4 T cells, B cells, dendritic cells and inflammatory macrophages in high MHC-I-expressing tumors, consistent with a more inflamed phenotype and intact antigen processing, presentation, and adaptive immunity. Consistent with our prior finding that BCs with high MHC-I neoantigens appear to downregulate MHC-I gene 
expression, the first negative association cluster showed that tumors with more abundant neoantigens often were associated with poor DC cell activation hallmarks (negative cluster 1) and low expression of MHC-I genes (negative cluster 2). Thus, one possible inference of these data is that basal-like BCs adapt to higher TMB and neoantigen abundance via downregulation of MHC-I, facilitating immune escape.

Given the finding of $H L A-A$ loss in the metastatic setting, we also sought to determine whether and how frequently this might occur in early-stage disease, by evaluating TCGA-BRCA data that contains RNAseq, DNAseq, and DNA methylation data ${ }^{30}$. Of 761 TCGA-BRCA tumors tested, 68 showed methylation of HLA-A and 8 showed methylation of 123 (Extended Data Fig.8a-c). Primary tumor $H L A-A$ methylation was associated with lower $H L A-A$ mRNA levels and lower expression of multiple adaptive immunity signatures (Extended Data Fig.8d-f). Importantly, tumors with $H L A-A$ methylation showed a worse survival outcome, even in multivariate analyses adjusting for stage and PAM50 subtype (Extended Data Fig.8g, h).

\section{Epigenetic suppression of cell adhesion in metastases}

We conducted a systematic analysis of DNA methylation changes associated with metastasis to uncover additional genes affected by an epigenetic mechanism. Cellular composition has a profound impact on DNA methylation profiles, thus different metastatic sites could produce false-positive results through contaminating stromal DNA methylation signals. We circumvented this metastatic site contamination problem by screening for loss of methylation in metastatic tumors at cis-regulatory elements that are consistently methylated in normal tissues representing the metastatic target tissues. We selected 19,607 CpG sites in distal enhancer-like elements defined by the ENCODE project ${ }^{31}$ that are constitutively methylated in eight normal tissue types. Supervised learning analyses comparing primary tumors versus metastases identified $123 \mathrm{CpG}$ sites that were significantly hypomethylated in metastatic tumors compared to their matched primaries. Using 11,348 ChIP-seq datasets, we found a significant overrepresentation of 47 DNA binding sites for 21 proteins at the 123 hypomethylated CpG sites (Fig. 4a). Proteins involved in estrogen signaling dominated binding at these hypomethylated $\mathrm{CpGs}$, including those encoded by ESR1, FOXA1, TFAP2A, and TFAP2C. We further investigated the distal elements bound by ESRI and FOXAl by performing Gene Ontology (GO) enrichment analysis of putative target genes regulated by these elements (see methods) (Fig. 4b). We found that genes involved in the 
regulation of cell adhesion are frequently represented among the target genes (Fig. 5b). However, surprisingly, we found that distal element hypomethylation is significantly associated with reduced expression of these associated genes, suggestive of negative regulation of these genes by estrogen signaling (Fig. 4c-f). We confirmed the significant association between distal element hypomethylation and reduced expression for JAM3 and FOXF1 in TCGA breast cancers (data were not available for CARD11 and UNC13D) (Fig. 4g, h).

We conducted a similar screen for gain of methylation at promoters by selecting $\mathrm{CpG}$ sites that are constitutively unmethylated in normal tissues representing the metastatic target tissues. We identified metastasis-associated promoter DNA hypermethylation of three genes (JAM3, YBX3, and $S Y N D G 1$ ), one of which was also identified in the distal element DNA hypomethylation analysis (Extended Data Fig. 9 left and middle panels). Gene expression of all three genes was significantly lower in metastatic tumors than in the matched primaries, with this observation more pronounced in HER2E or Luminal subtypes (Extended Data Fig. 9c, f and i).

\section{Clonal evolution and subtype switching}

Many publications have studied DNA-based clonal evolution in longitudinal samples, and in response to therapeutic selection ${ }^{32,33}$. We focused here on three cases that showed gene expression-based subtype switching to address the question of whether this change in expression phenotype was accompanied by, and possibly driven by, DNA clonality changes (Fig. 5a-o). Patient AER8 was diagnosed with an ER+/PR+/HER2- Luminal A subtype primary tumor and received neoadjuvant chemotherapy and adjuvant endocrine therapy plus everolimus through a clinical trial (NCT01674140); she was diagnosed with liver metastases after $\sim 20$ months of treatment, received an additional 3 lines of therapy and then succumbed to disease, at which time biopsies of several metastatic lesions were obtained (Fig. 5a). The two assayed liver metastases showed clonal changes relative to the primary and were clonally related (Fig. 5b, c), a finding supported by the closely related cancer-associated DNA hypermethylation profiles observed in the two liver metastases (Fig. 5e). Metastasis M2 was assayed by RNAseq and showed a subtype switch to HER2E (yet remained clinically HER2-negative), with an increase in proliferation signature, and a decrease in HLA-A mRNA levels and immune cell features (Fig. 5d). Acquisition of the HER2E subtype in the absence of gain of HER2-amplification in metastatic 
samples has been reported ${ }^{3,16,34}$, but this is the first instance to demonstrate its' association with a DNA clonality change.

A second example of subtype switching with DNA clonality changes was patient AFR3, who was diagnosed with an ER+/PR+/HER2- Luminal A BC. She was treated with chemotherapy and then endocrine therapy and progressed with multiple metastases, of which the brain metastasis was surgically removed (Fig. 5f). The brain specimen showed a dramatic change to ER-/PR/HER2+, and gene expression analysis confirmed a subtype switch to HER2E (Fig. 5f), with a concomitant DNA clonality change that included acquisition of HER2 amplification and an ERBB2/HER2 E668Q activating mutation (Fig. 5g, h). This DNA clonal change was also reflected in phenotypic diversity of the DNA hypermethylation landscape between primary and metastasis (Fig. 5j), and was associated with a downregulation of ESR 1 and $P G R$ and upregulation of $E R B B 2$ gene expression (Fig. 5i).

In contrast to patient AFR3 whose BC switched to HER2E subtype likely due in part to an acquired HER2 amplification, patient AFE4 showed a reverse trend. Namely, this patient presented with an ER+/PR+/HER2+ BC (HER2E expression subtype), where it was noted that the HER2 IHC result was 2+/FISH inconclusive but was HERmark assay positive. After 30 months of HER2-directed therapy with trastuzumab, tumor progression was documented, a lung biopsy was obtained, and the clinical receptor status remeasured, indicating an ER+/HER2status. Additional treatments were given, however, the tumor progressed and the patient died 18 months later. At autopsy, multiple metastatic tumor specimens were obtained (Fig. 5k). Interestingly, the 3 metastatic specimens assayed by RNAseq showed subtype switches to Luminal A or Luminal B, DNA clonality changes and loss of HER2 amplification (Fig. 51, m), while HER2 mRNA levels were slightly decreased (Fig. 5n); thus, in this case the primary tumor showed clinically significant HER2 levels, HER2-directed therapy was given, and the therapeutic pressure presumably resulted in loss of HER2 at the DNA level. DNA methylation features largely agreed with the DNA clonal evolution in this case except for right lung metastasis (M1) that presented with the lowest DNA tumor purity score (Fig. 5o).

\section{Discussion}


Established metastatic tumors are extremely challenging to treat, and their biology is complex. Overall, when primary tumors are compared to their matched metastases, the dominant genomic patterns seen in the primary tends to be maintained in the metastasis, however, significant differences have been identified which may contribute to the poor prognosis associated with MBC. In performing multi-platform analyses of primary tumors versus metastases, we discovered several key patterns that explain some metastatic tumor behaviors, including events derived from epigenetic, genomic, and transcriptomic evolution.

A key epigenetic mechanism identified here was DNA methylation of $H L A-A$, typically in basallike/TNBC metastatic disease, leading to lower expression of $H L A-A$ (often in high neoantigen burden tumors) and associated lowered expression of multiple immune cell features. This loss of $H L A-A$ mRNA also was observed in TCGA primary cancers at a lower frequency than in our metastatic cohort, and when observed, also was linked to lower immune cell features and a worse overall survival. These findings provide a molecular explanation for the loss of immune cell features in some metastatic tumors, which has potential therapeutic implications. One such implication is that ICIs may have little effect on these $H L A-A$ low tumors due to loss of antigen presentation protein coding genes that appear to be coordinately regulated with $H L A-A$. In this setting, these tumors will evade the immune system's continual surveillance for tumor neoantigens (noting these HLA-A methylated tumors tend to have high neoantigen burdens). These results also suggest a biomarker driven therapeutic approach wherein $H L A-A$ DNA methylated tumors (i.e. the biomarker) could be targeted with DNA de-methylating drugs, in combination with immune checkpoint inhibitors ${ }^{35}$. Clearly, preclinical and prospective testing will be needed to test this biomarker-driven therapeutic hypothesis.

Changes in the somatic genetics of metastatic breast tumors are well documented ${ }^{2,3}$, and here we extend the changes seen in metastatic tumors into the epigenetic landscape (i.e., DNA methylation and gene expression features). In 1/3 of the patients, we identified a gene expression-defined tumor subtype switch, especially frequent in luminal/ER+ patients, wherein these gene expression changes were mirrored by DNA clonality and methylation changes.

In addition to possible epigenetic changes in tumor cells, RNAseq analysis of multiple immune cell signatures showed dramatic differences simply according to site of metastasis. It is already 
appreciated that the brain is an immune privileged site ${ }^{36}$, and our results confirm this finding. There is also growing evidence that the liver is similarly immune privileged ${ }^{37}$, and our results confirm low immune cell features in liver metastases. Using this unique resource, we found that in 9/15 patients with multiple metastases, liver metastases had the lowest immune cell features of any synchronous site of metastasis. These comparative metastatic tissue site findings have immediate clinical implications because the liver is a relatively accessible and biopsied site for metastatic evaluation, and our data suggests that liver metastases are more likely to have low immune cell features, which may bias assay results of immune therapy biomarker positivity.

Interestingly, we discovered through systematic screening for metastasis-associated DNA methylation changes, mechanisms leading to downregulation of JAM3 expression in metastatic tumors, namely DNA hypomethylation at a distal ESRI binding site and DNA hypermethylation of the gene promoter. Notably, it has been reported that JAM2 overexpression (a second junctional adhesion molecule (JAM) family member) in breast cancer cell lines blocks invasion and migration ${ }^{38}$, and JAM3 is silenced by DNA hypermethylation in colorectal cancers and its suppression promotes cell migration in cell lines ${ }^{39}$. In addition, a causal interaction between DNA methylation and ER-mediated repression of gene expression has been previously reported $^{40}$, and our finding that multiple genes encoding proteins regulating cell adhesion appear to be negatively regulated by estrogen signaling may have functional consequences for progression to metastasis. This is consistent with prior reports of estrogen-mediated downregulation of E-cadherin in breast cancer cells ${ }^{41}$.

Lastly, our three examples of clonal evolution highlight DNA clonality shifts coincident with gene expression-based subtype changes. In patient AER8 the clonal shift and altered expression subtype did not include any new actionable mutations, which may represent the most common finding with respect to changes in DNA-based actionable mutations in the metastatic setting ${ }^{42}$. In patient AFR3, an actionable variant was identified (i.e., gain of HER2), and trastuzumab therapy was given, although the tumor progressed. Patient AFE4 highlights yet another challenge of precision medicine wherein an actionable DNA-based feature is identified and targeted (i.e., HER2-amplification) yet the tumor eventually evades the treatment by deleting the therapeutic target. Each of these patients illustrates a third clinical impact of this study; if medically possible, biopsy and characterize the metastatic disease as it has likely changed relative to the primary 
tumor, possibly on the DNA level, the protein level (ER/PR/HER2), the tumor epigenetic phenotype (intrinsic subtype), and/or in the tumor microenvironment (immune cell frequencies).

There are limitations to this study, most notably the sample size that precluded statistical analyses of somatic mutation frequencies in primary tumors versus metastases. A second challenge was the integration of data from fresh frozen specimens with FFPE specimens. Nonetheless, we identified many multi-platform supported findings concerning tumor clonal evolution and immune evasion that are common in MBCs. These multi-platform genomic data of metastatic disease presented here are highly complementary to the TCGA resource of primary disease $^{30,43,44}$, and have already begun to illuminate the molecular features of the metastatic landscape that may impact the care of breast cancer patients.

Accession numbers and data sharing: All newly generated data is under submission to the dbGAP and GEO and should be completed by the time of publication. All of the resources used during this manuscript are summarized in a table in the methodology section.

Acknowledgements. The Aurora US Metastatic Breast Cancer Project is funded by the Breast Cancer Research Foundation (Grant ID:ELFF-14-002) through the Evelyn H. Lauder Founder's Fund for Metastatic Breast Cancer Research. We next acknowledge the many patients and their families for their selfless donations of specimens for this project and the collaboration of numerous patient advocate representatives. Additional support was provided by many institution's infrastructures support including the RedCap instance used to capture clinical data in this project was supported by the National Center for Advancing Translational Sciences (NCATS), National Institutes of Health, through Grant Award Number UL1TR002489. This work was also supported by Vanderbilt-Ingram Cancer Center Support Grant P30 CA68485 and NCI SPORE 2P50CA098131-17 (JMB), Lineberger Comprehensive Cancer Center Grant P30CA016086-45 and NCI Breast SPORE program P50-CA58223 (CMP, LAC). The results published here are in whole or part based upon data from the Cancer Genome Atlas managed by the NCI and NHGRI (dbGaP accession phs000178). The content is solely the responsibility of the authors and does not necessarily represent the official views of the NIH. 


\section{Author contributions}

The AURORA Metastatic Project consortium contributed collectively to this study.

Biospecimens were provided by tissue medical centers in the USA based upon the infrastructure of the Translational Breast Cancer Research Consortium and processed by a Biospecimens Core Resource (Nationwide Children's Hospital). Data generation and analyses were performed by a genome sequencing center (Nationwide Children's Hospital), two cancer genome characterization centers (Van Andel Institute and Univ. of North Carolina), and multiple genome data analysis centers. All data were released through the Data Coordinating Center (Univ. of Pittsburgh). We also acknowledge the following AURORA's investigators for their author contributions including: AURORA Steering Committee (SGH, LN, ALR, WFS, ACW, IK, NED, LAC, TAK, CMP), Clinical Working Group (ACGC, ALG, CA, MCL, AW, SA, KAH, TAK, BHP, IK, MFR, RN, NUL, CI, PKM, AMS, MCL), Pathology Working Group (ALR, WFS), Molecular Working Group (SGR, TH, GLW, BJK, AGC, TP, AADC, YX, MBMC, AR, EK, MAS, CF, CJC, RTB, JSP, KKF, HS, FJC, UC, MD, JS, AR, ALR, AVL, JMB, KAH, PWL, ERM, CMP), and the Biospecimens Working Group (JB, KL, SP, JGF). All authors reviewed, contributed to the writing of the manuscript, and approved this submission.

\section{Conflicts of Interest.}

The following authors disclose COIs including CMP has: listed as an inventor on patent applications on the Breast PAM50 assay; equity stock holder and consultant of BioClassifier LLC. JSP is listed as an inventor on patent applications on the Breast PAM50 assay. HS has authorship and equity in AnchorDX, authorship with Illumina, and IP license with TruDiagnostics Inc. BHP has: Royalties: Horizon Discovery, LTD; Consultant: EQRx, Sermonix, Hologics, Jackson Laboratories, Guardant Health Inc; Unpaid consultant: Tempus; Consultant and ownership interest: Celcuity; Research Contracts: GE Healthcare, Lilly and Pfizer. IK has: Consulting Fees (e.g. advisory boards); Author; Bristol Meyers Squibb, Daiichi/Sankyo, Macrogenics, Context Therapeutics, Taiho Oncology, Genentech/Roche, Seattle Genetics. Contracted Research (NOTE: Pls must provide disclosure in this category); Author; Genentech/Roche, Pfizer. Other; Author; Novartis, Merck (DSMB member). CA has: Royalty; Author; UpToDate, Jones and Bartlett. Consulting Fees (e.g. advisory boards); Author; PUMA (Start 9/2018 - 9/2019), Genentech (1/2019-), Eisai (1/2019-), IPSEN (2/2019-). Contracted 
Research (NOTE: Pls must provide disclosure in this category); Author; PUMA, Lily, Merck, Seattle Genetics, Nektar, Tesaro, G1-Therapeutics. MFR has: Consulting Fees (e.g. advisory boards); Author; Macrogenics, Daiichi, and Genentech. Contracted Research (NOTE: Pls must provide disclosure in this category); Author; Pfizer. RN has: Consulting Fees (e.g. advisory boards); Author; Aduro, AstraZeneca, Athenex, Celgene, Daiichi Sankyo, Inc, Genentech, MacroGenics, Merck, Novartis, Pfizer, Puma, Syndax. Contracted Research (NOTE: Pls must provide disclosure in this category); Author; AstraZeneca, Celgene, Concept Therapeutics, Genentech/Roche, Immunomedics, Merck, Odonate Therapeutics, Pfizer, Seattle Genetics. Other; Author; DSMB:G1 Therapeutics; Steering Committee: OBI Pharm, Inc. NUL has: Consulting Fees (e.g. advisory boards); Author; Seattle genetics, puma and daichii. Contracted Research (NOTE: Pls must provide disclosure in this category); Author; Genentech, Seattle Genetics, Pfizer. CI has Consulting Fees (e.g. advisory boards); Author; Pfizer, AstraZeneca, Genentech, Novartis and Puma. Fees for Non-CME Services Received Directly from Commercial Interest or their Agents (e.g. speakers' bureaus); Author; Genentech. MCL has: Author; Eisai, Genentech, GRAIL, Janssen, Merck, Novartis, Seattle Genetics, Tesaro. JMB has: Receipt of Intellectual Property Rights / Patent Holder; Author; Provisional patents regarding immunotherapy targets and biomarkers in cancer. Consulting Fees (e.g. advisory boards); Author; Novartis. Contracted Research (NOTE: Pls must provide disclosure in this category); Author; Genentech/Roche, Bristol Myers Squibb, and Incyte Corporation. PWL has: Consulting Fees (e.g. advisory boards); Progenity, Inc., Stock Options; Author; AnchorDx, Author: Progenity, Inc.Illumina, Inc., IP License; TruDiagnostic Inc. All remaining authors have no relevant disclosures. 
497 Fig. 1. Study design and global genomic patterns of metastatic breast tumors. a. Eligibility criteria and cohort description of the AURORA Metastatic Project. b. Diagram of the shared or individual tumor DNA methylation, whole genome sequencing (WGS)/whole exome sequencing (WES) and RNA sequencing data successfully performed on each the 55 patients of AURORA MBC cohort. c. Global profiling of DNA methylation landscape using the top 5000 most variable hypermethylated CpGs in 99 paired and 34 unpaired primary and metastatic tumors. d. Supervised hierarchical cluster analysis of 102 paired and 21 unpaired primary and metastatic RNA sequenced tumors using 1710 intrinsic subtype gene list. e. OncoPrint panel of DNA somatic mutations displaying 36 most frequency mutated genes in 41 primary and 93 metastatic tumors. The percentage on the left indicates the mutation frequency of each gene across samples. Del, deletion; Ins, insertion.

Fig. 2. Subtype switching and supervised analysis of gene expression signatures between primary and metastatic tumors. a. Overall molecular intrinsic subtype change between 39 patient-matched primary breast and 1 or more metastatic tumors. b. Patient-specific molecular subtype changes in 39 patient-matched primary breast and 1 or more metastatic tumors. c. Heatmap of the significantly different signatures between primary and metastatic tumors, in Luminal/HER2E and d) basal-like subtype only. Significance of the differences between primary and metastasis was calculated using linear mixed models $(q<0.01)$. Significant signatures are row ordered from high to low according to $\beta$-coefficients (or regression coefficients) and divided according to upregulated (positive) or downregulated (negative) in metastasis. Patients are column ordered according to PAM50 molecular subtype and divided according to primary and metastasis. Signatures scores were calculated in the Level 4 RNAseq data (see methods). Normal-like tumors and post-treatment primaries were removed from the analysis in the AURORA cohort.

Fig. 3. $H L A-A$ dysregulation and impact on immune-related features in metastatic tumors. a. Hypermethylated $\mathrm{CpG}$ sites in $H L A-A$ (8 CpG sites), HLA-B (14 CpG sites) and HLA-C (12 CpG sites) of 133 primary and metastatic tumors. b. HLA-A, HLA-B, HLA-C and HLA-DRB5 focal deletions in HLA region of 49 patients. c. Heatmap representation of the difference in $H L A-A,-B,-C, B 2 M$ and TAPBP gene expression values, and GP2-Immune-Metagene and Hallmark IFN $\gamma$ response gene signature scores, calculated between paired primary $(n=36)$ and metastatic $(n=60)$ tumors. Normal-like paired and unpaired tumors were removed from this analysis (Paired Normal and unpaired group from the "Pairs-PAM50-Prim" column of the supplementary table 2). Gene and signature scores are ordered according to $H L A-A$ gene expression changes. For the 60 metastases, association is shown with $H L A-A,-B,-C$, methylation/DNA focal deletion status and $B 2 M$ and TAPBP methylation status, PAM50 and site of metastasis. d. Left panel, MHC-I-associated neoantigens levels in MHC-I altered (HLA-A, - B, 
- $C$ hypermethylation or focal deletion)) versus others (non-altered) in Basal-like tumors when data was available ( $\mathrm{n}=25,5$ primary and 20 metastasis). Right panel, TMB in MHC-I altered versus others in Basal-like tumors when data was available $(n=35,11$ primary and 24 metastasis). e. Left panel, MHC-I-associated neoantigens levels in MHC-I versus others in Luminal/HER2E tumors when data was available ( $\mathrm{n}=44,10$ primary and 34 metastasis). Right panel, TMB in MHC-I altered versus others in Luminal/HER2E tumors when data was available ( $\mathrm{n}=66,22$ primary and 44 metastasis). f. $H L A-A,-B,-C$ and $B 2 M$ gene expression values are shown in HLA-A versus others of 17 Basal-like primary and 25 metastatic tumors. g. MHC-I metagene signature scores according to lines of therapies in metastatic samples $(\mathrm{N}=77)$. $\mathbf{h}$. MHCI metagene signature score differences between primary and metastatic tumors according to molecular subtype in AURORA $(n=61)$ and RAP $(n=75)$ cohorts. i. Correlation matrix and unsupervised hierarchical clustering of CIBERSORTx-based immune-cell scores in Basal-like samples ( $\mathrm{n}=42,17$ primary and 25 Metastasis). Positive clusters (PC1 and PC2) and negative clusters (NC1 and NC2) reflects the highest or lowest correlated immune-related signatures scores per CIBERSORTx. Correlation was measured using the Pearson correlation coefficient and $\mathrm{p}$ values $<0.05$ are shown as $(*)$. Comparisons between 2 paired groups were performed by $\mathrm{t}-$ test. Comparison between more than 2 groups was performed by ANOVA with post hoc Tukey's test. Box-and-whisker plots display the median value on each bar, showing the lower and upper quartile range of the data and data outliers. The whiskers represent the interquartile range. Statistically significant values are highlighted in red or indicated by *. Each mark represents the value of a single sample. ns, non-significant.

\section{Fig. 4. Metastatic tumor-associated DNA hypomethylation at distal enhancer elements. a,} Analysis of DNA binding proteins at the significantly hypomethylated $\mathrm{CpG}$ sites. Each dot represents one of the 11,348 ChiP-seq data analyzed. Y-axis represents the odds ratio of enrichment, and $\mathrm{X}$-axis represents the number of significant $\mathrm{CpGs}$ overlapping protein binding sites. The size of the dot denotes the statistical significance of the enrichment (Fisher's exact test). b. Gene Ontology (GO) analysis of putative target genes for the hypomethylated ESRI or FOXA1 distal binding sites. Shown are the top fifty GO terms based on the $\mathrm{P}$ values from Fisher's exact test. Dots sizes are proportional to the number of genes. Red text highlights cell adhesion GO terms and genes of interest. c-f. Analysis of putative enhancer target genes involved in the regulation of cell adhesion. For each gene, a comparison of distal element DNA methylation between primary and metastatic tumors is shown on the left, and putative target gene expression between methylated $(\beta$-value $\geq 0.4)$ vs. unmethylated $(\beta$-value of $<0.4)$ tumors is shown on the right. The $P$ values were calculated using Welch's two-sample $t$-test. $\mathbf{g}, \mathbf{h}$. Analysis of distal element DNA hypomethylation (left) and putative target gene expression (right) in TCGA breast cancer data. Normal breast tissue samples are indicated in dark gray, and tumor samples are color-coded by the PAM50 molecular subtype. The samples were grouped into either methylated or unmethylated using a $\beta$-value threshold of 0.4 . The $P$ values were calculated using Welch's two-sample t-test. 
577 Fig. 5. Multi-omics patient characterization of individual AURORA cases. Timeline of

578 patient clinical history, clonal structure, clonal evolution, transcriptome, and methylome

579 description of patient AER8 (a, b, c, d, e), AFR3 (f, g. g, i, j) and AFE4 (k, l, m, n, o).

580 Transcriptome data reflects gene expression values and gene expression signatures calculated in

581 using normalized RNAseq data. P, primary; M, metastasis, LumA, luminal A, LumB, luminal B;

582 HyperMe, hypermethylation.

583

584 
Supplementary Table 1. Clinical and pathologic demographics of the AURORA US patient cohort.

Extended Data Fig.1. Survival outcomes according to clinical subtypes of AURORA cohort. used to study the overall survival from breast cancer diagnosis ("First Primary Receptor at diagnosis" column of Supplementary table 2) in HER2 positive (HER2+), Hormone receptor positive and HER2 negative (HR+/HER2-) and TNBC (triple negative breast cancers). $\mathbf{b}$. Kaplan-Meier, the log rank test and Cox proportional hazards regression model to study the overall survival from metastatic breast cancer diagnosis ("Metastasis original receptors" column of Supplementary table 2) in HER2+, HR+/HER2- and TNBC. In absence of HR/HER2 status in the metastatic relapse we used the data from the most recent biopsy. EBC, early breast cancer; MBC, metastatic breast cancer; confidence interval (CI). Statistically significant values are highlighted in red.

601

Extended Data Fig.2. Clinical subtype and molecular subtype distribution according to site of metastasis. a. Distribution of the 55 diagnosed primary tumors by clinical receptor status (TNBC, ER+/HER2-, HER2+, and unknown, left side) linked to their anatomic sites of metastasis (right). Clinical receptor status at the time of first primary diagnosis ("First Primary Receptors" column of Supplementary table 2). b. Distribution of 39 diagnosed primary tumors by gene expression based intrinsic molecular subtype when available (left) linked to their anatomic sites of metastasis (right).

609

Extended Data Fig.3. Correlation between tumor cellularity metrics and immune signatures. a. Supervised hierarchical clustering of the top 1,000 leukocyte-specifically methylated probes and the bottom 1,000 tumor tissue-specifically methylated probes, after ranking all probes based on the mean leukocytes - mean tumor tissues. For the 133 tumors, association is shown with tumor type, tumor purity, and estimated leukocyte fraction scores ${ }^{19,20}$. b. Pearson correlation between the difference (Metastasis - Primary gene expression values) of the Leukocyte fraction scores and GP2-Immune-Metagene signature scores (calculated from the Level 4 RNAseq data). Higher scores mean higher expression in metastasis compared to primary tumors. Correlation was measured using the Pearson correlation coefficient. c. Spearman correlations between GP2-Immune-Metagene signature scores and several pathology determined scores (\% Tumor nuclei, \% of normal cells, \% of Stromal cells, \% Lymphocyte infiltration and

621 tumor cellularity) or genomic scores (Estimate-Stromal scores, Estimate-Immune Score and 
Estimate Score using ESTIMATE method ${ }^{45}$. Rho (spearman correlation coefficient, $\rho$ ). d. Pearson correlation of GP2-Immune-Metagene signature score and \% of Tumor nuclei form pathology report. Statistically significant values are highlighted in red. GP2-Immune-Metagene signature scores were calculated from the Level 4 RNAseq data (see methods).

\section{Extended Data Fig.4. Supervised analysis of gene expression signatures according to site of} metastasis in AURORA or combined AURORA-RAP-GEICAM cohorts. a. Heatmap depicting the differentially expressed (DE) signatures between primary $(n=26)$ and metastasis $(n=69)$ in the AURORA cohort using all samples. b. Heatmap depicting the DE signatures between paired primary $(n=5)$ and brain metastasis $(n=5)$ in the AURORA cohort. c. Heatmap depicting the DE signatures between paired primary $(n=6)$ and liver metastasis $(n=6)$ in the AURORA cohort. d. Heatmap depicting the DE signatures between Basal-like paired primary $(n=5)$ and brain metastasis $(n=11)$ in the AURORA-RAP-GEICAM cohort. d. Heatmap depicting the DE signatures between Luminals (LumA, LumB, and HER2E) paired primary $(\mathrm{n}=22)$ and liver metastasis $(\mathrm{n}=25)$ in the AURORA-RAP-GEICAM cohort. Significance of the differences between primary and metastasis was calculated using linear mixed models $(q<0.01)$. Significant signatures are row ordered from high to low according to $\beta$-coefficients (or regression coefficients) and divided according to upregulated (positive) or downregulated (negative) in metastasis. Patients are column ordered according to PAM50 molecular subtype and divided according to primary and metastasis. Signatures scores were calculated in the Level 4 RNAseq data (see methods). Normal-like tumors and post-treatment primaries were removed from the analysis in the AURORA cohort.

\section{Extended Data Fig.5. Patients with multiple metastases examined for immune features in} AURORA-RAP combined cohort. a. gene expression signature scores of GP2-ImmuneMetagene are shown according to individual specimens coming from patients with at least 2 metastases analyzed by RNAseq data $(n=16)$. The star indicates liver specimens with the lowest expression of signature. b. Signature expression changes between paired primary and liver, brain, lung or rest of metastasis of GP2-Immune-Metagene signature divided by Basal-like or Luminal groups (Luminal A, B and HER2E). Comparisons between 2 paired groups were performed by paired, 2-tailed t-test Statistically significant values are highlighted in red. Brt, breast; Adr, adrenal; Liv, liver; Dip, Diaphragm; Per, peritoneum; Rct, rectum, Skn, skin; Stm, stomach; Thy, Thyroid; SoftT, soft tissue; LN, lymph node; Ple, pleura; Lun, lung, Brn, brain; Bon, bone; Kid, kidney; Che, chest; Spl, spleen; Mes, mesentery; Pan, pancreas; LumA, luminal A; LumB, luminal B.

\section{Extended Data Fig.6. Difference in $H L A-A$ and immune-signature expression between} primary and metastatic tumors. a. Waterfall plot of AURORA cases showing the difference 
between primary $(n=36)$ and metastasis $(n=60)$ (Difference: Metastasis - Primary gene expression value) ordered from the highest (left) to the lowest (right) signature score for $H L A-A$ mRNA expression (bottom panel). The top panel show the difference of primary versus metastases for GP2-Immune-Metagene values (top panel). Yellow stars highlight $H L A-A$ Hypermethylated cases and green stars highlight the samples with DNA HLA-A focal deletions. b. Waterfall plot of RAP cases showing the difference between primary $(n=15)$ and metastasis $(\mathrm{n}=56)$ (Difference: Metastasis - Primary gene expression value) ordered from the highest (left) to the lowest (right) signature score for HLA-A mRNA expression (bottom panel). The top panel show the difference of primary versus metastases for GP2-Immune-Metagene values (top panel). Pairs with a Normal-like primary tumor were removed from the analysis. LumA, luminal A; LumB, luminal B.

Extended Data Fig.7. $H L A-A$ gene expression correlation with number of neoantigens. a. Linear relationship between number of neoantigens and $H L A-A,-B$ and $C$ gene expression Level 4 RNAseq data (see methods) of Basal-like only primary and metastatic tumors. Correlation was measured using the Pearson correlation coefficient. Statistically significant values are highlighted in red. b. Violin plots showing changes in gene expression for $H L A-A,-B$, and -C between primary and metastatic samples (Difference: Metastasis - Primary gene expression values) in Basals and Luminals/HER2E metastatic tumors. c. Patient-specific changes in gene expression for $H L A-A,-B$, and $-C$ between primary and metastatic samples (Difference: Metastasis - Primary gene expression values) in Basal-likes ( $\mathrm{n}=24$ metastasis) and Luminals/HER2E ( $\mathrm{n}=34$ metastasis) metastatic tumors of AURORA cohort. Normal-like paired and unpaired tumors were removed from this analysis (Paired Normal and unpaired group from the "Pairs-PAM50-Prim" column of the Supplementary table 2).

\section{Extended Data Fig.8. $H L A-A$ methylated primary tumors and prognostic value of $\boldsymbol{H L A}-\boldsymbol{A}$} in TCGA data. a. Oncoprint diagram depicting $H L A-A$ and $H L A-B$ methylated cases using 761 primary tumors of TCGA BRCA dataset according to PAM50 molecular subtype. b. Proportion of each molecular subtypes found in HLA-A (68) and HLA-B (8) methylated tumors. c. Hypermethylated CpG sites in $H L A-A$ ( 9 CpG sites) using 761 TCGA primary breast tumors and 74 tumor-adjacent breast tissues. d. Boxplots of $H L A-A$ mRNA gene expression levels according to DNA methylation status. Comparisons between 2 paired groups were performed by t-test. e. Scatter plot showing the correlation between $H L A-A$ mRNA expression values and DNA methylation levels ( $\beta$-values). f. Boxplots of gene expression signature B cell/T cell cooperation and IgG scores according to DNA methylation status in tumors and tumor-adjacent breast tissues in TCGA-BRCA. Box-and-whisker plots display the median value on each bar, showing the lower and upper quartile range of the data and data outliers. The whiskers represent the interquartile range. Comparison between 2 groups was performed by ANOVA with post hoc Tukey's test. Statistically significant values are highlighted in red. Each mark represents the 
value of a single sample. g. Kaplan-Meier plots using the log rank test of overall survival from primary tumors according to $H L A-A$ methylation status. h. Multivariable Cox proportional hazards analyses of TCGA BRCA patients for overall survival prediction using the covariates of $H L A-A$ methylation status, PAM50 subtypes and tumor stage (10 Stage IV patients were removed from the analysis). Hazard ratio $(\mathrm{HR})=1$ : no effect. $\mathrm{HR}<1$ : reduction in hazard. $\mathrm{HR}>$ 1: increase in hazard. Statistically significant values are highlighted in red. Unme, unmethlylated; HyperMe, hypermethylated.

\section{Extended Data Fig. 9. DNA methylation alterations associated with metastatic tumors. a-i.} Analysis of metastasis-associated promoter DNA hypermethylation of three genes (JAM3, YBX3 and SYNDIG1) encoding components of tight junctions or regulation of adhesion molecules. For each gene, a comparison of promoter CpG DNA methylation between primary and metastatic tumors is shown on the left $(\mathrm{a}, \mathrm{d}, \mathrm{g})$, a second comparison of promoter CpG DNA methylation between (1) unmethylated primaries $(\beta$-value of $<0.3)$ and their paired metastasis and (2) methylated primaries ( $\beta$-value of $>0.3$ ) with their paired metastasis $(b, c, e)$ is shown in the middle, and a third comparison of gene expression between primary and metastatic tumors based on all samples (All), Luminal A-B and HER2E only (Luminals/HER2E), and basal-like subtype only (Basals) is shown on the right (c, f, i). P, primary; M, metastasis; P-Unme, Unmethylated primary; $\mathrm{P}-\mathrm{Me}$, Methylated primary. 
7351 Siegel, R. L., Miller, K. D., Fuchs, H. E. \& Jemal, A. Cancer Statistics, 2021. CA Cancer J Clin

71, 7-33, doi:10.3322/caac.21654 (2021).

2 Bertucci, F. et al. Genomic characterization of metastatic breast cancers. Nature 569, 560-564, doi:10.1038/s41586-019-1056-z (2019).

3 Aftimos, P. et al. Genomic and transcriptomic analyses of breast cancer primaries and matched metastases in AURORA, the Breast International Group (BIG) molecular screening initiative. Cancer discovery, doi:10.1158/2159-8290.CD-20-1647 (2021).

$4 \quad$ Paul, M. R. et al. Genomic landscape of metastatic breast cancer identifies preferentially dysregulated pathways and targets. J Clin Invest 130, 4252-4265, doi:10.1172/JCI129941 (2020).

$5 \quad$ Finn, R. S. et al. Palbociclib and Letrozole in Advanced Breast Cancer. N Engl J Med 375, 19251936, doi:10.1056/NEJMoa1607303 (2016).

$6 \mathrm{Im}$, S. A. et al. Overall Survival with Ribociclib plus Endocrine Therapy in Breast Cancer. $N$ Engl J Med 381, 307-316, doi:10.1056/NEJMoa1903765 (2019).

7 Goetz, M. P. et al. MONARCH 3: Abemaciclib As Initial Therapy for Advanced Breast Cancer. $J$ Clin Oncol 35, 3638-3646, doi:10.1200/JCO.2017.75.6155 (2017).

8 Modi, S. et al. Trastuzumab Deruxtecan in Previously Treated HER2-Positive Breast Cancer. $N$ Engl J Med 382, 610-621, doi:10.1056/NEJMoa1914510 (2020).

9 Murthy, R. K. et al. Tucatinib, Trastuzumab, and Capecitabine for HER2-Positive Metastatic Breast Cancer. N Engl J Med 382, 597-609, doi:10.1056/NEJMoa1914609 (2020).

10 Schmid, P. et al. Pembrolizumab for Early Triple-Negative Breast Cancer. N Engl J Med 382, 810-821, doi:10.1056/NEJMoa1910549 (2020).

11 Schmid, P. et al. Atezolizumab and Nab-Paclitaxel in Advanced Triple-Negative Breast Cancer. N Engl J Med 379, 2108-2121, doi:10.1056/NEJMoa1809615 (2018).

12 Cortes, J. et al. Pembrolizumab plus chemotherapy versus placebo plus chemotherapy for previously untreated locally recurrent inoperable or metastatic triple-negative breast cancer (KEYNOTE-355): a randomised, placebo-controlled, double-blind, phase 3 clinical trial. Lancet 396, 1817-1828, doi:10.1016/S0140-6736(20)32531-9 (2020).

13 Miles, D. et al. Primary results from IMpassion131, a double-blind, placebo-controlled, randomised phase III trial of first-line paclitaxel with or without atezolizumab for unresectable locally advanced/metastatic triple-negative breast cancer. Ann Oncol 32, 994-1004, doi:10.1016/j.annonc.2021.05.801 (2021).

14 Network, T. C. G. A. R. \& Perou, C. M. The Cancer Genome Atlas Pan-Cancer analysis project. Nat Genet 45, 1113-1120, doi:10.1038/ng.2764 (2013).

15 Parker, J. S. et al. Supervised Risk Predictor of Breast Cancer Based on Intrinsic Subtypes. J Clin Oncol, doi:JCO.2008.18.1370 [pii]

\subsection{0/JCO.2008.18.1370 (2009).}

16 Garcia-Recio, S. et al. FGFR4 regulates tumor subtype differentiation in luminal breast cancer and metastatic disease. J Clin Invest, doi:10.1172/JCI130323 (2020).

17 Siegel, M. B. et al. Integrated RNA and DNA sequencing reveals early drivers of metastatic breast cancer. J Clin Invest 128, 1371-1383, doi:10.1172/JCI96153 (2018).

18 Fernandez-Martinez, A. et al. Survival, Pathologic Response, and Genomics in CALGB 40601 (Alliance), a Neoadjuvant Phase III Trial of Paclitaxel-Trastuzumab With or Without Lapatinib in HER2-Positive Breast Cancer. J Clin Oncol, JCO2001276, doi:10.1200/JCO.20.01276 (2020).

19 Carter, S. L. et al. Absolute quantification of somatic DNA alterations in human cancer. Nat Biotechnol 30, 413-421, doi:10.1038/nbt.2203 (2012). 
20 Thorsson, V. et al. The Immune Landscape of Cancer. Immunity 48, 812-830 e814, doi:10.1016/j.immuni.2018.03.023 (2018).

21 Subramanian, A. et al. Gene set enrichment analysis: a knowledge-based approach for interpreting genome-wide expression profiles. Proc Natl Acad Sci U S A 102, 15545-15550 (2005).

22 Iglesia, M. D. et al. Genomic Analysis of Immune Cell Infiltrates Across 11 Tumor Types. J Natl Cancer Inst 108, doi:10.1093/jnci/djw144 (2016).

23 Iglesia, M. D. et al. Prognostic B-Cell Signatures using mRNA-Seq in Patients with SubtypeSpecific Breast and Ovarian Cancer. Clin Cancer Res 20, 3818-3829, doi:10.1158/10780432.CCR-13-3368 (2014).

24 Newman, A. M. et al. Robust enumeration of cell subsets from tissue expression profiles. Nat Methods 12, 453-457, doi:10.1038/nmeth.3337 (2015).

25 Fan, C. et al. Building prognostic models for breast cancer patients using clinical variables and hundreds of gene expression signatures. BMC Med Genomics 4, 3, doi:1755-8794-4-3 [pii]

10.1186/1755-8794-4-3 (2011).

26 Hollern, D. P. et al. B Cells and T Follicular Helper Cells Mediate Response to Checkpoint Inhibitors in High Mutation Burden Mouse Models of Breast Cancer. Cell 179, 1191-1206 e1121, doi:10.1016/j.cell.2019.10.028 (2019).

27 Bhattacharya, A., Hamilton, A. M., Troester, M. A. \& Love, M. I. DeCompress: tissue compartment deconvolution of targeted mRNA expression panels using compressed sensing. Nucleic Acids Res 49, e48, doi:10.1093/nar/gkab031 (2021).

28 Harrell, J. C. et al. Genomic analysis identifies unique signatures predictive of brain, lung, and liver relapse. Breast Cancer Res Treat 132, 523-535, doi:10.1007/s10549-011-1619-7 (2012).

29 Newman, A. M. et al. Determining cell type abundance and expression from bulk tissues with digital cytometry. Nat Biotechnol 37, 773-782, doi:10.1038/s41587-019-0114-2 (2019).

30 Ciriello, G. et al. Comprehensive Molecular Portraits of Invasive Lobular Breast Cancer. Cell 163, 506-519, doi:10.1016/j.cell.2015.09.033 (2015).

31 Consortium, E. P. et al. Expanded encyclopaedias of DNA elements in the human and mouse genomes. Nature 583, 699-710, doi:10.1038/s41586-020-2493-4 (2020).

32 Nik-Zainal, S. et al. Landscape of somatic mutations in 560 breast cancer whole-genome sequences. Nature 534, 47-54, doi:10.1038/nature17676 (2016).

33 Stephens, P. J. et al. The landscape of cancer genes and mutational processes in breast cancer. Nature 486, 400-404, doi:10.1038/nature11017 (2012).

34 Jorgensen, C. L. T. et al. PAM50 Intrinsic Subtype Profiles in Primary and Metastatic Breast Cancer Show a Significant Shift toward More Aggressive Subtypes with Prognostic Implications. Cancers (Basel) 13, doi:10.3390/cancers13071592 (2021).

35 Topper, M. J., Vaz, M., Marrone, K. A., Brahmer, J. R. \& Baylin, S. B. The emerging role of epigenetic therapeutics in immuno-oncology. Nature reviews. Clinical oncology 17, 75-90, doi:10.1038/s41571-019-0266-5 (2020).

36 Achrol, A. S. et al. Brain metastases. Nat Rev Dis Primers 5, 5, doi:10.1038/s41572-018-0055-y (2019).

37 Lee, J. C. et al. The Liver-Immunity Nexus and Cancer Immunotherapy. Clin Cancer Res, doi:10.1158/1078-0432.CCR-21-1193 (2021).

38 Peng, Y. et al. JAM2 predicts a good prognosis and inhibits invasion and migration by suppressing EMT pathway in breast cancer. Int Immunopharmacol 103, 108430, doi:10.1016/j.intimp.2021.108430 (2021).

39 Zhou, D., Tang, W., Zhang, Y. \& An, H. X. JAM3 functions as a novel tumor suppressor and is inactivated by DNA methylation in colorectal cancer. Cancer Manag Res 11, 2457-2470, doi:10.2147/CMAR.S189937 (2019).

40 Pathiraja, T. N. et al. Epigenetic reprogramming of HOXC10 in endocrine-resistant breast cancer. Science translational medicine 6, 229ra241, doi:10.1126/scitranslmed.3008326 (2014). 
$83141 \quad$ Oesterreich, S. et al. Estrogen-mediated down-regulation of E-cadherin in breast cancer cells.

$832 \quad$ Cancer Res 63, 5203-5208 (2003).

83342 van de Haar, J. et al. Limited evolution of the actionable metastatic cancer genome under therapeutic pressure. Nat Med 27, 1553-1563, doi:10.1038/s41591-021-01448-w (2021).

43 Thennavan, A. et al. Molecular analysis of TCGA breast cancer histologic types. Cell Genomics 1, doi:10.1016/j.xgen.2021.100067 (2021).

44 Network, T. C. G. A. R. \& Perou, C. M. Comprehensive molecular portraits of human breast tumours. Nature 490, 61-70, doi:10.1038/nature11412 (2012).

45 Yoshihara, K. et al. Inferring tumour purity and stromal and immune cell admixture from 


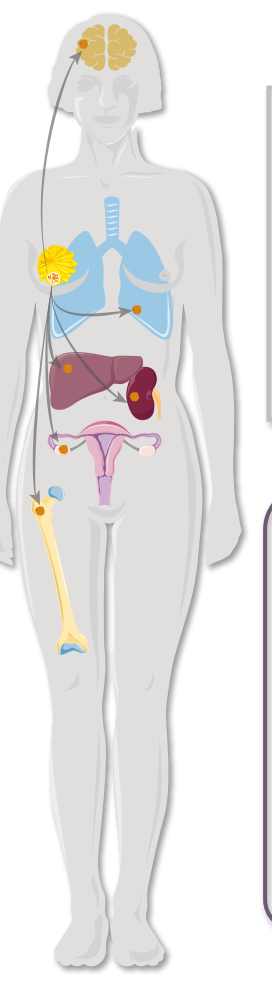

C

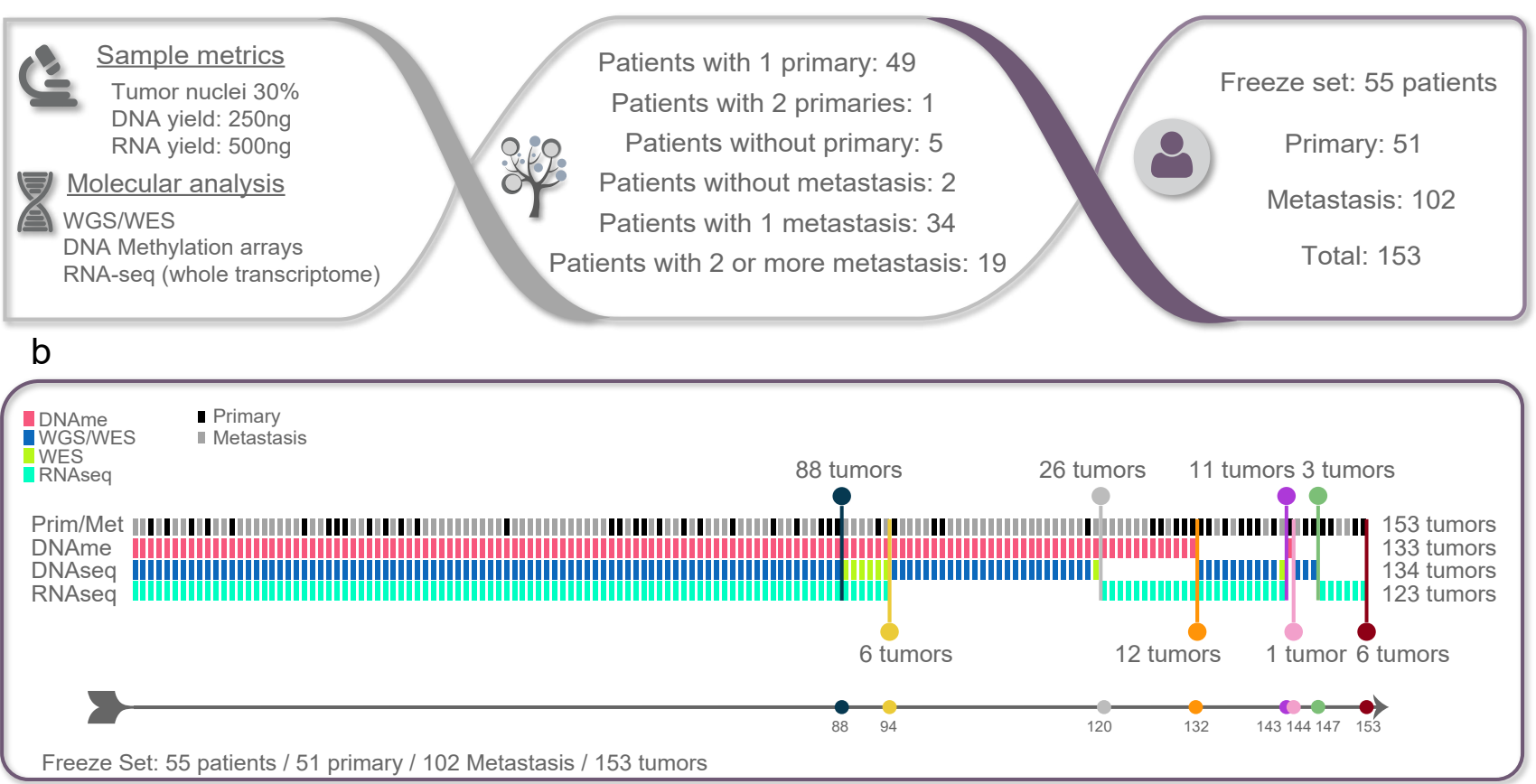

DNA methylation data

d

Tumor type
Site Metastases Tumor purity

Tissue Typ
PAM55
Patient

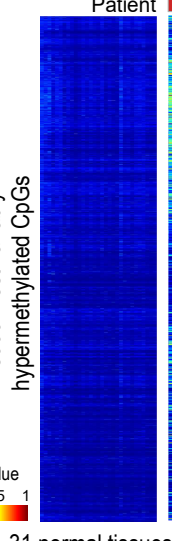

31 normal tissues (8 tissue types)

Tumor type

- Primary $\square$ Metastasis

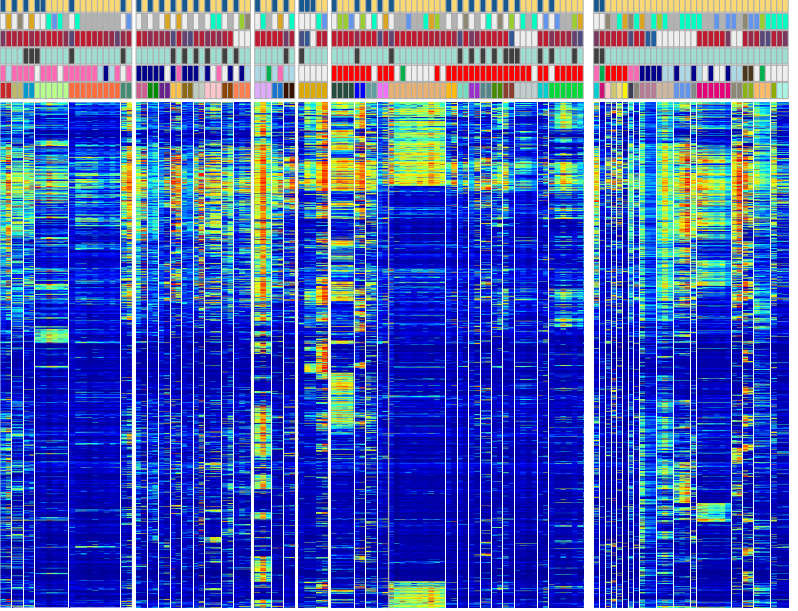

36 primary and 63 paired metastasis (33 patients - 99 Paired tumors)

Site of Metastases

$\square$ Brain $\square$ Liver $\square$ Lung $\square$ Lymph node $\square$ Others $\square$ NA (Primary)
Tissue Type FF 口FFPE
RNA sequencing data

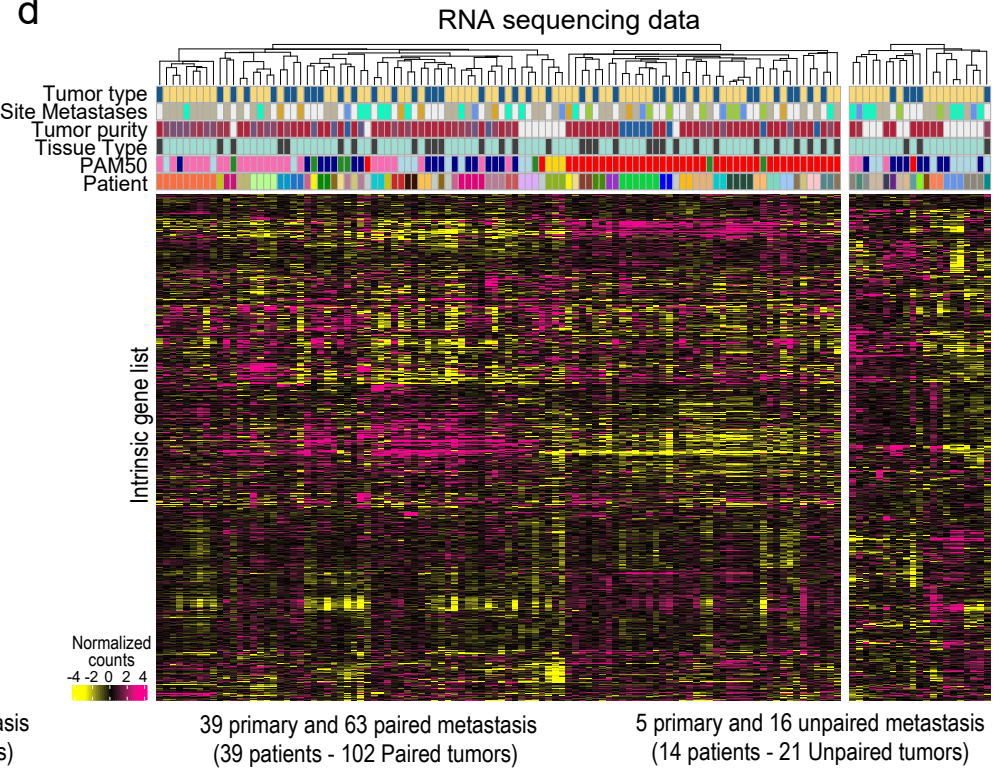

PAM50

Tumor Purity

(DNASeq-base

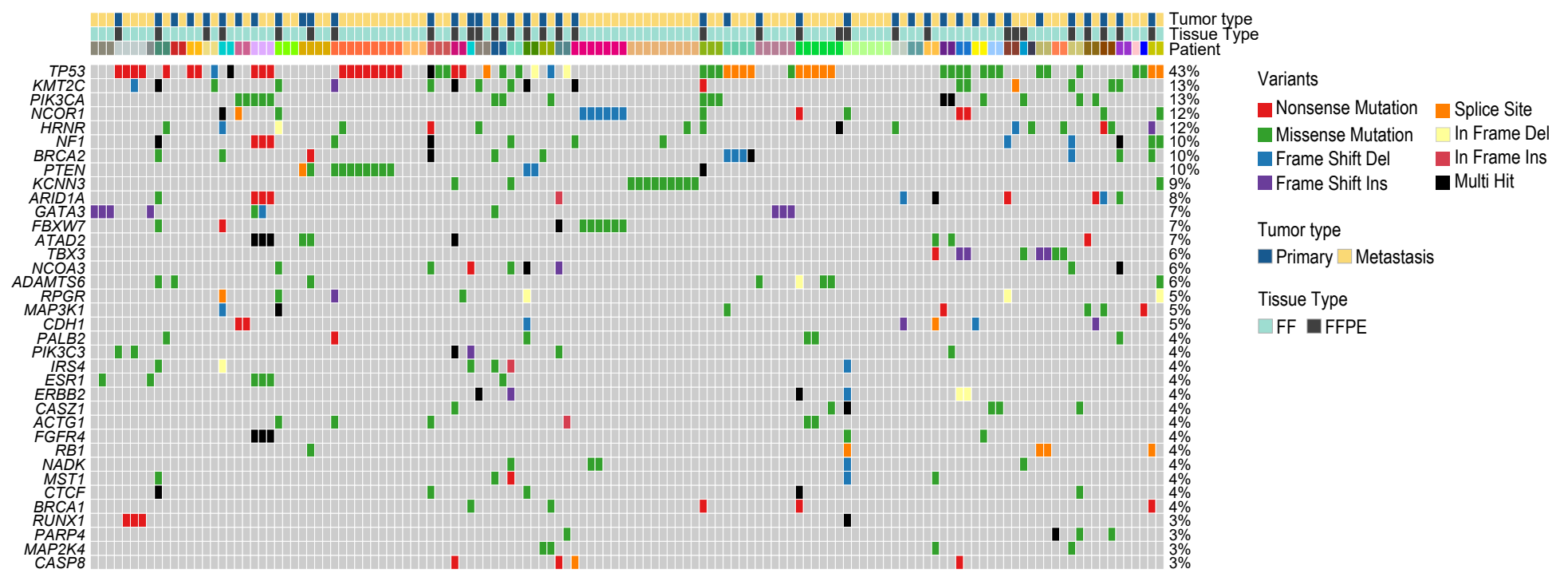


Fig. 1. Study design and global genomic patterns of metastatic breast tumors. a. Eligibility criteria and cohort description of the AURORA Metastatic Project. b. Diagram of the shared or individual tumor DNA methylation, whole genome sequencing (WGS)/whole exome sequencing (WES) and RNA sequencing data successfully performed on each the 55 patients of AURORA MBC cohort. c. Global profiling of DNA methylation landscape using the top 5000 most variable hypermethylated CpGs in 99 paired and 34 unpaired primary and metastatic tumors. d.

Supervised hierarchical cluster analysis of 102 paired and 21 unpaired primary and metastatic RNA sequenced tumors using 1710 intrinsic subtype gene list. e. OncoPrint panel of DNA somatic mutations displaying 36 most frequency mutated genes in 41 primary and 93 metastatic tumors. The percentage on the left indicates the mutation frequency of each gene across samples. Del, deletion; Ins, insertion. 
a

\begin{tabular}{|c|c|c|c|c|c|c|c|}
\hline \multirow[b]{2}{*}{ Primary } & \multicolumn{6}{|c|}{ Metastasis } & \multirow[b]{2}{*}{ Patients } \\
\hline & Claudin & Basal & HER2E & LumA & LumB & Normal & \\
\hline Claudin & $2(100 \%)$ & 0 & 0 & 0 & 0 & 0 & 1 \\
\hline Basal & 0 & $22(92 \%)$ & ) 0 & 0 & 0 & $2(8 \%)$ & 16 \\
\hline HER2E & 0 & 0 & $12(63 \%)$ & $3(16 \%)$ & $4(21 \%)$ & 0 & 8 \\
\hline LumA & 0 & $1(9 \%)$ & $2(18 \%)$ & $6(51 \%)$ & $1(9 \%)$ & $1(9 \%)$ & 8 \\
\hline LumB & 0 & 0 & $1(25 \%)$ & 0 & $2(50 \%)$ & $1(25 \%)$ & 3 \\
\hline Normal & 0 & $2(67 \%)$ & ) $1(33 \%)$ & 0 & 0 & 0 & 3 \\
\hline
\end{tabular}

C

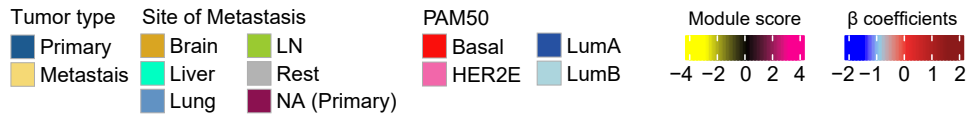

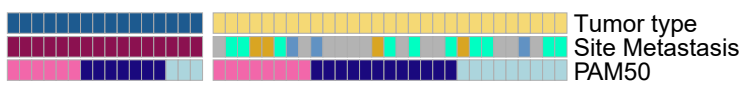
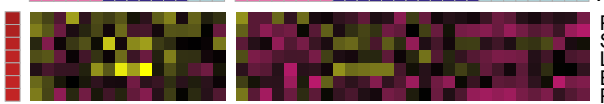

Pcorr Hypoxia Low Correlation

ums HER2E UP metastatic signature

Lums HER2E UP metastatic
Euclidean Distance 2 Clow
Pcorr IGS Correlation
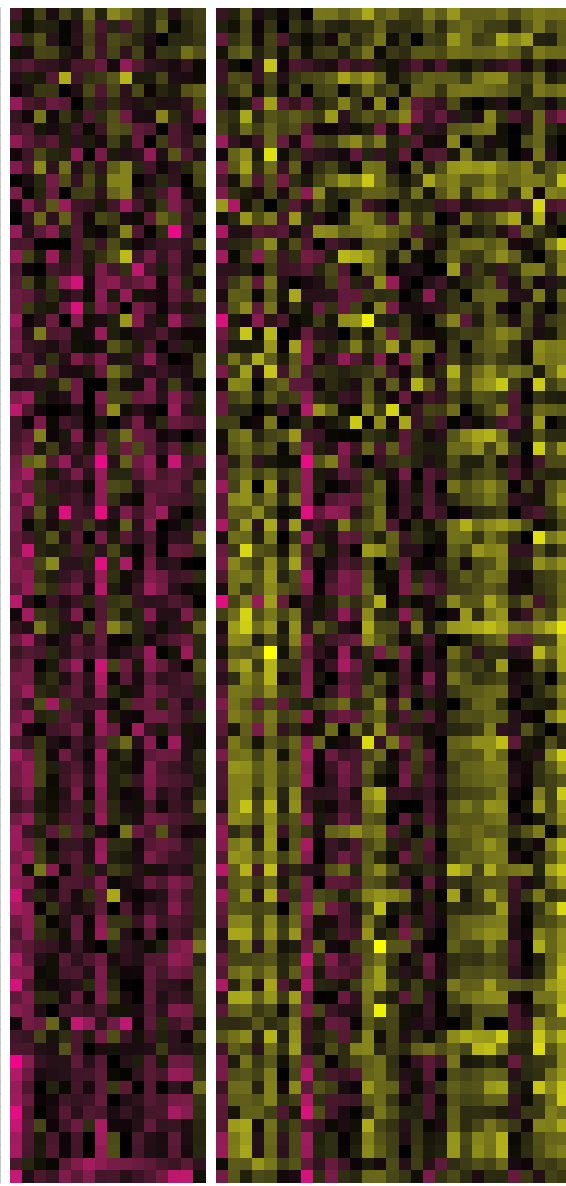

Vascular Content

VEGF 13genes

Pcorr Hypoxia High Correlation

Verhaak Stromal

Fibromatosis

Fibroblast Cluster

im2009 Stroma

TCGA.BRCA.1198 COLLAGEN11A

Lums HER2E DOWN metastatic signature

AMPH EPIREGULIN Cluster

GSEA GP15 EGF signailng

TNBC Clinically Relevant

TCGA.BRCA.1198 BASAL

BASAL Cluster

GSEA GP17 Basal signaling

GSEA GP6 Squamous differentiation development

GSEA GP21 Anti apoptosis DNA stability

REPLICATION STRESS POS

MASC Up

MS CD44 UP

MECM_BMC

MBASAL

EMT UP Weinberg

MET DOWN Significant Genes LOW BASALS 1

GSEA GP4 MES ECM

Bcells Centrocyte

Duke Module20 stat3

Duke Module21 tgfb

IMMUNE Bindea Cell Lymph vessels

IMMUNE Bindea Cell Neutrophils

MDSC Neutrophil Youn

MDSC tumor MO Schlecker

Pcorr squamoid

PDGFRB Single Gene

FOS JUN Cluste

TCGA.BRCA.1198 immune FOS JUN IL6

TCGA TGFB score b

Primary

39 patients

Figure 2

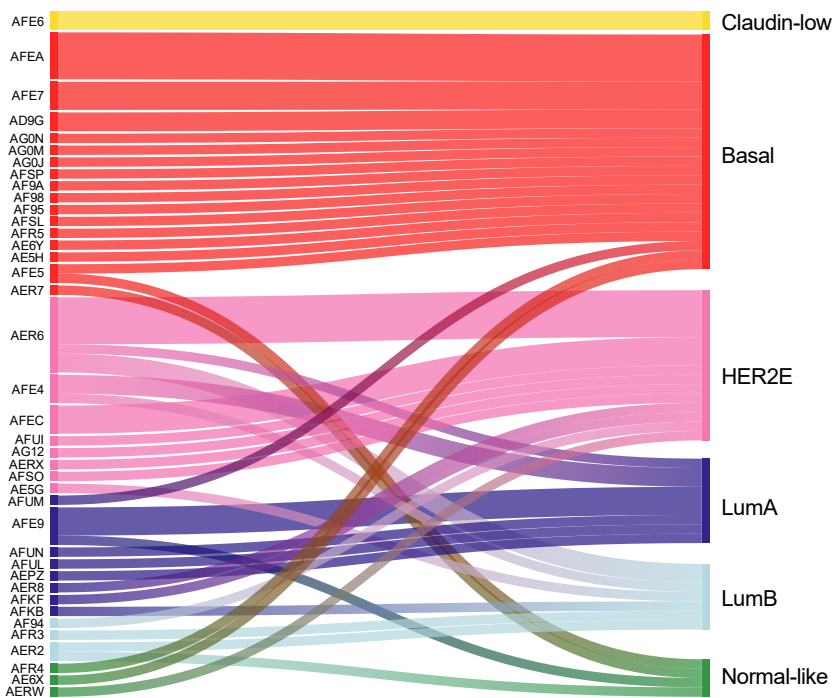

d

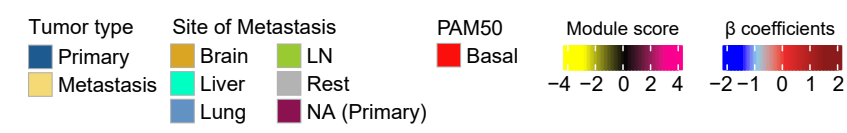
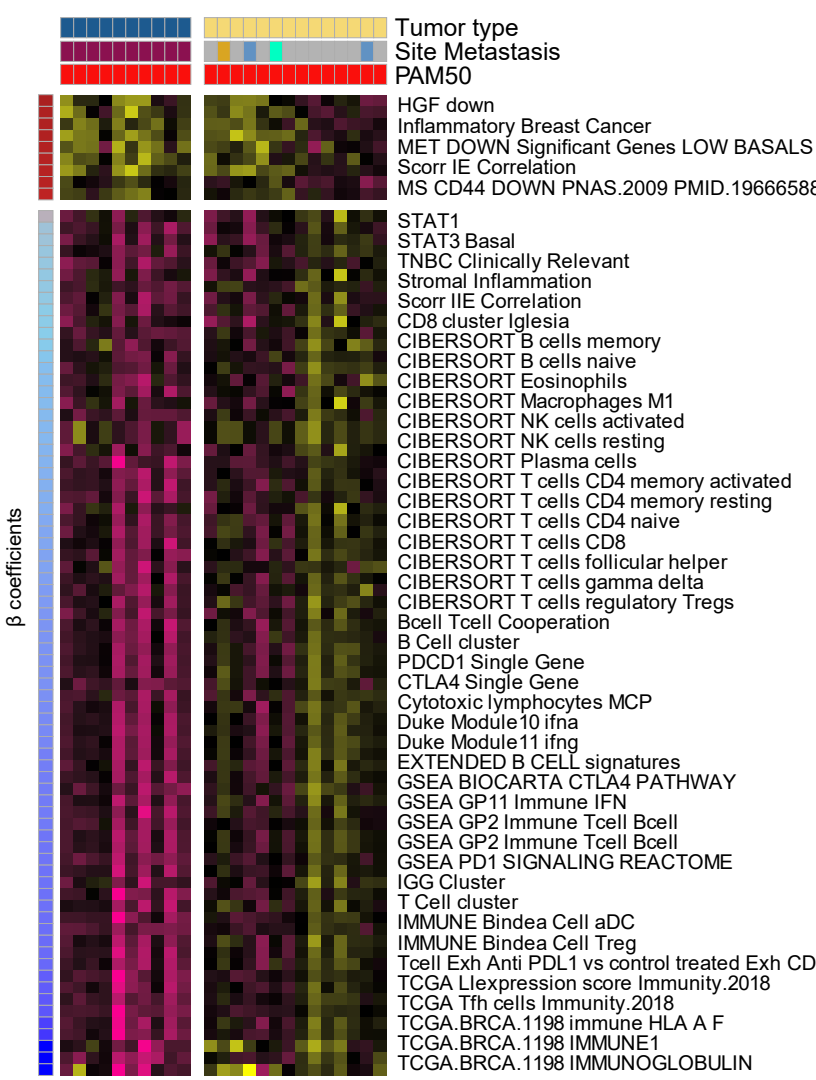

STAT1 1
STAT3 Basal

TNAT3 Basal

Stromal Inflammation

CD8 cluster Iglesia

CIBERSORT B cells memory

CIBERSORT B cells naive

CIBERSORT Macrophages M1

CIBERSORT NK cells activated

CIBERSORT NK cells resting

CBERSORT Plasma cells

CIBERSORT T cells CD4 memory activate

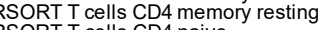

CIBERSORT T cells CD8

CIBERSORT T cells follicular helper

CIBERSORT T cells gamma delta

CIBERSORT T cells regulatory Tregs

B Cell cluster

PDCD1 Single Gene

Cytotoxic lymphocytes MCP

Duke Module 10 ifna

Duke Module 11 ifng

GSEA BIOCARTA CTLA4 PATHWAY

GSEA GP11 Immune IFN

GSEA GP2 Immune Tcell Bcell

GSEA PD1 SIGNALING REACTOME

IGG Cluster

IMMUNE Bindea Cell aDC

Biter

TCGA Llexpression score Immunity.2018

.

CGA.BRCA. 1198 immune HLA A F

TCGA.BRCA. 1198 IMMUNE1
TCGA.BRCA.1198 IMMUNOGLOBULIN 
Fig. 2. Subtype switching and supervised analysis of gene expression signatures between primary and metastatic tumors. a. Overall molecular intrinsic subtype change between 39 patient-matched primary breast and 1 or more metastatic tumors. b. Patient-specific molecular subtype changes in 39 patient-matched primary breast and 1 or more metastatic tumors. c. Heatmap of the significantly different signatures between primary and metastatic tumors, in Luminal/HER2E and d) basal-like subtype only. Significance of the differences between primary and metastasis was calculated using linear mixed models $(\mathrm{q}<0.01)$. Significant signatures are row ordered from high to low according to $\beta$-coefficients (or regression coefficients) and divided according to upregulated (positive) or downregulated (negative) in metastasis. Patients are column ordered according to PAM50 molecular subtype and divided according to primary and metastasis. Signatures scores were calculated in the Level 4 RNAseq data (see methods). Normal-like tumors and post-treatment primaries were removed from the analysis in the AURORA cohort. 


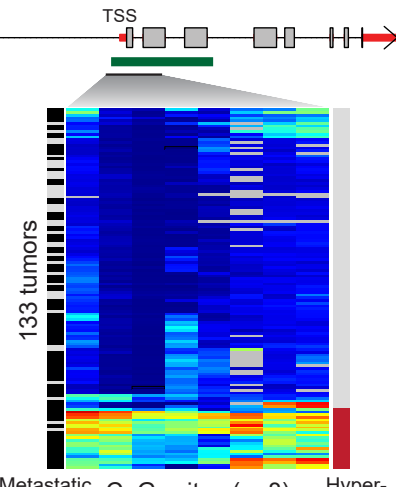

Metastatic $\mathrm{CpGs}$ sites $(\mathrm{n}=8) \quad \begin{gathered}\text { Hyper- } \\ \text { tumor }\end{gathered}$ \begin{tabular}{lclll}
$\begin{array}{l}\text { Non-detection } \\
\text { probe masking }\end{array}$ & \multicolumn{2}{|c|}{$\beta$-value } & CpG island
\end{tabular} C

AURORA 36 patients - 60 metastasis b
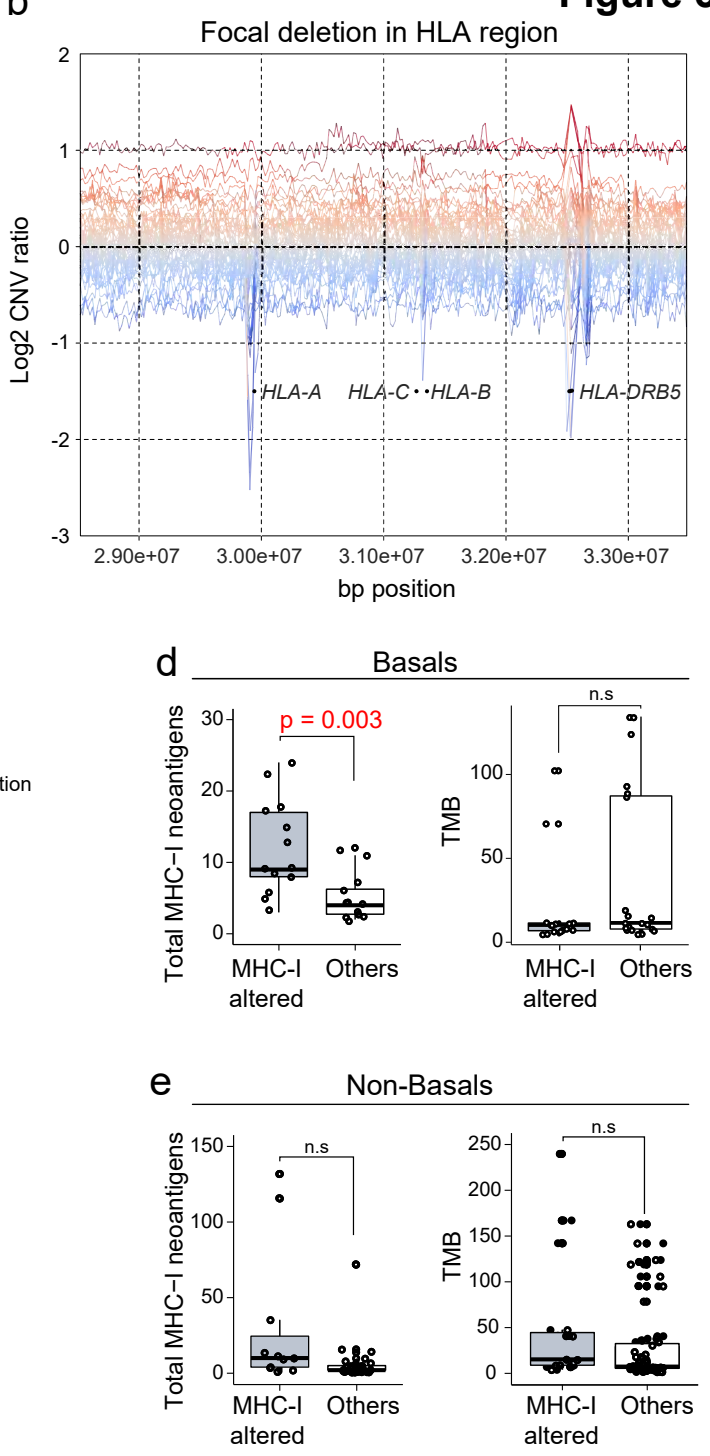

f
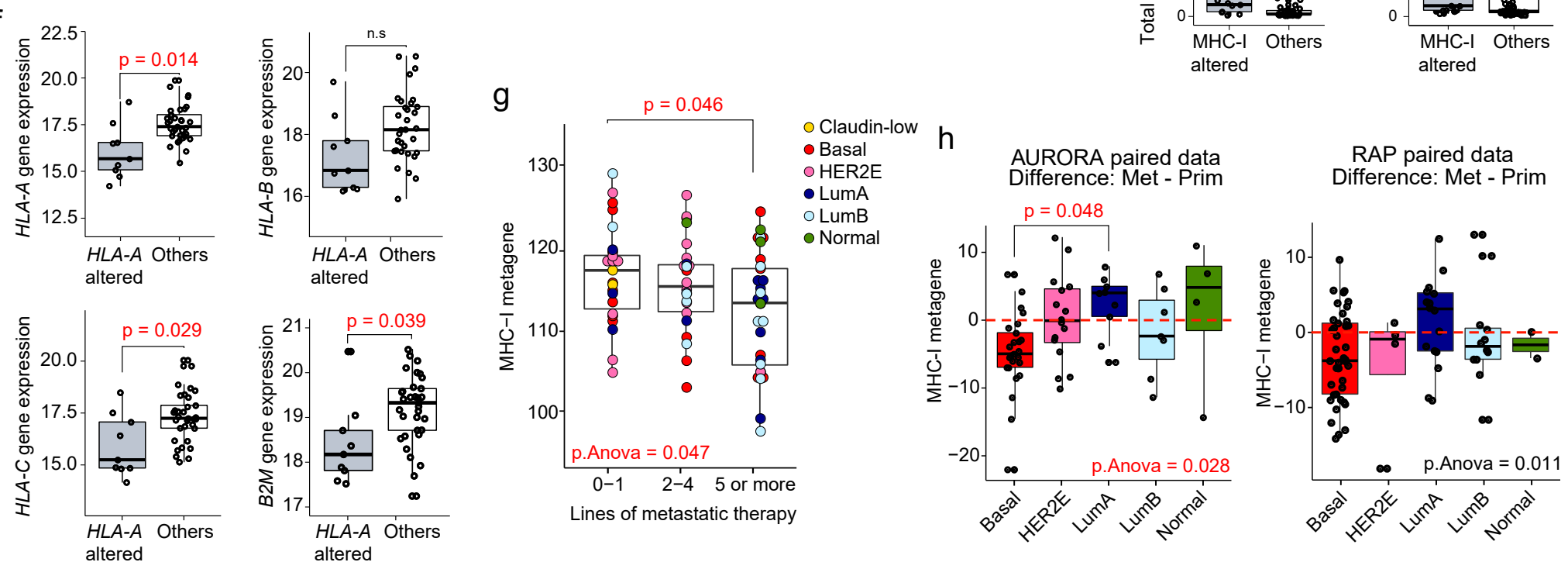

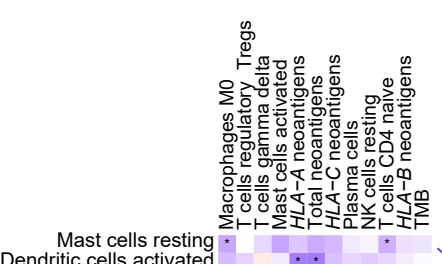

Mast cells resting
ditic cells activated NK cells activated
Macrophages M2
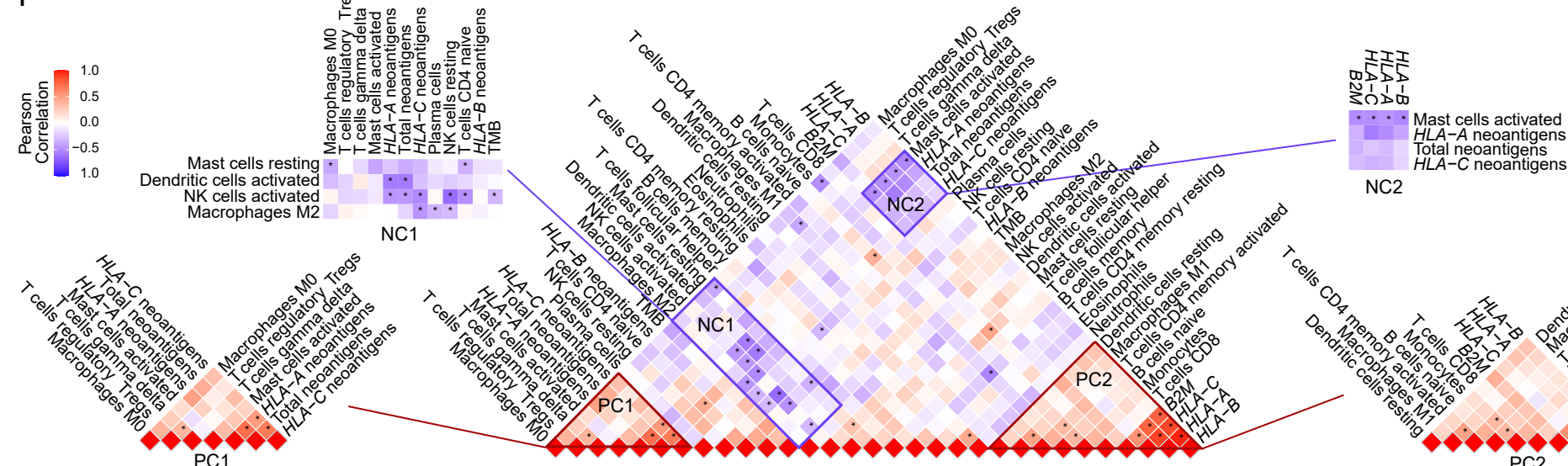
Fig. 3. $\boldsymbol{H L A}-\boldsymbol{A}$ dysregulation and impact on immune-related features in metastatic tumors. a. Hypermethylated $\mathrm{CpG}$ sites in $H L A-A$ ( $8 \mathrm{CpG}$ sites), $H L A-B$ (14 CpG sites) and HLA-C (12 CpG sites) of 133 primary and metastatic tumors. b. HLA-A, HLA-B, HLA-C and HLA-DRB5 focal deletions in HLA region of 49 patients. c. Heatmap representation of the difference in $H L A-A,-B,-C, B 2 M$ and TAPBP gene expression values, and GP2-Immune-Metagene and Hallmark IFN $\gamma$ response gene signature scores, calculated between paired primary $(\mathrm{n}=36)$ and metastatic $(n=60)$ tumors. Normal-like paired and unpaired tumors were removed from this analysis (Paired Normal and unpaired group from the "Pairs-PAM50-Prim" column of the supplementary table 2). Gene and signature scores are ordered according to $H L A-A$ gene expression changes. For the 60 metastases, association is shown with $H L A-A,-B,-C$, methylation/DNA focal deletion status and B2M and TAPBP methylation status, PAM50 and site of metastasis. d. Left panel, MHC-I-associated neoantigens levels in MHC-I altered (HLA-A, $-B$, $-C$ hypermethylation or focal deletion)) versus others (non-altered) in Basal-like tumors when data was available ( $\mathrm{n}=25,5$ primary and 20 metastasis). Right panel, TMB in MHC-I altered versus others in Basal-like tumors when data was available ( $\mathrm{n}=35,11$ primary and 24 metastasis). e. Left panel, MHC-I-associated neoantigens levels in MHC-I versus others in Luminal/HER2E tumors when data was available ( $\mathrm{n}=44,10$ primary and 34 metastasis). Right panel, TMB in MHC-I altered versus others in Luminal/HER2E tumors when data was available ( $\mathrm{n}=66,22$ primary and 44 metastasis). f. $H L A-A,-B,-C$ and $B 2 M$ gene expression values are shown in $H L A-A$ versus others of 17 Basal-like primary and 25 metastatic tumors. g. MHC-I metagene signature scores according to lines of therapies in metastatic samples $(\mathrm{N}=77)$. h. MHCI metagene signature score differences between primary and metastatic tumors according to molecular subtype in AURORA $(\mathrm{n}=61)$ and RAP $(\mathrm{n}=75)$ cohorts. i. Correlation matrix and unsupervised hierarchical clustering of CIBERSORTx-based immune-cell scores in Basal-like samples ( $\mathrm{n}=42,17$ primary and 25 Metastasis). Positive clusters (PC1 and $\mathrm{PC} 2)$ and negative clusters ( $\mathrm{NC1}$ and $\mathrm{NC} 2$ ) reflects the highest or lowest correlated immune-related signatures scores per CIBERSORTx. Correlation was measured using the Pearson correlation coefficient and $\mathrm{p}$ values $<0.05$ are shown as $(*)$. Comparisons between 2 paired groups were performed by $\mathrm{t}$ test. Comparison between more than 2 groups was performed by ANOVA with post hoc Tukey's test. Box-and-whisker plots display the median value on each bar, showing the lower and upper quartile range of the data and data outliers. The whiskers represent the interquartile range. Statistically significant values are highlighted in red or indicated by *. Each mark represents the value of a single sample. ns, non-significant. 
a

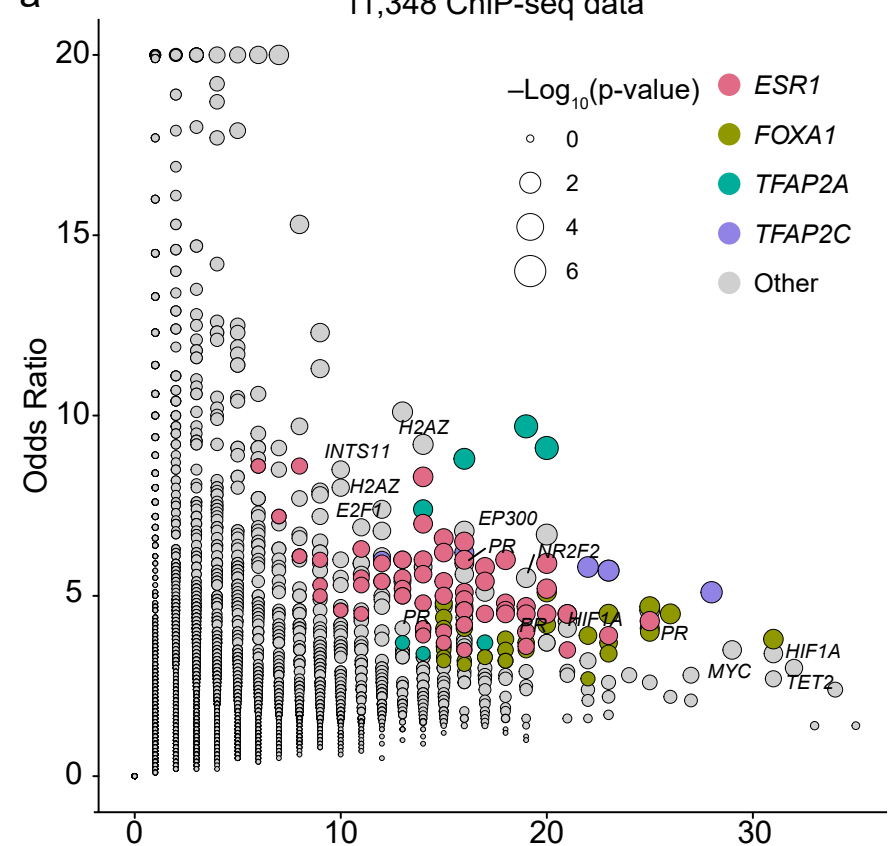

Number of overlapping hypomethylated probes
C

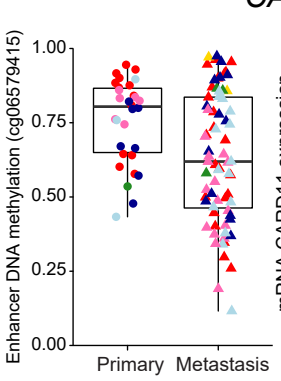

e

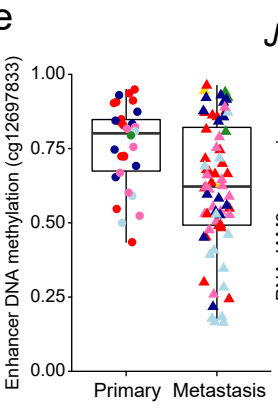

d

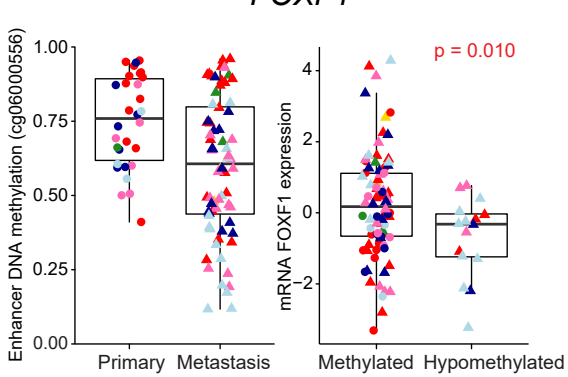

b

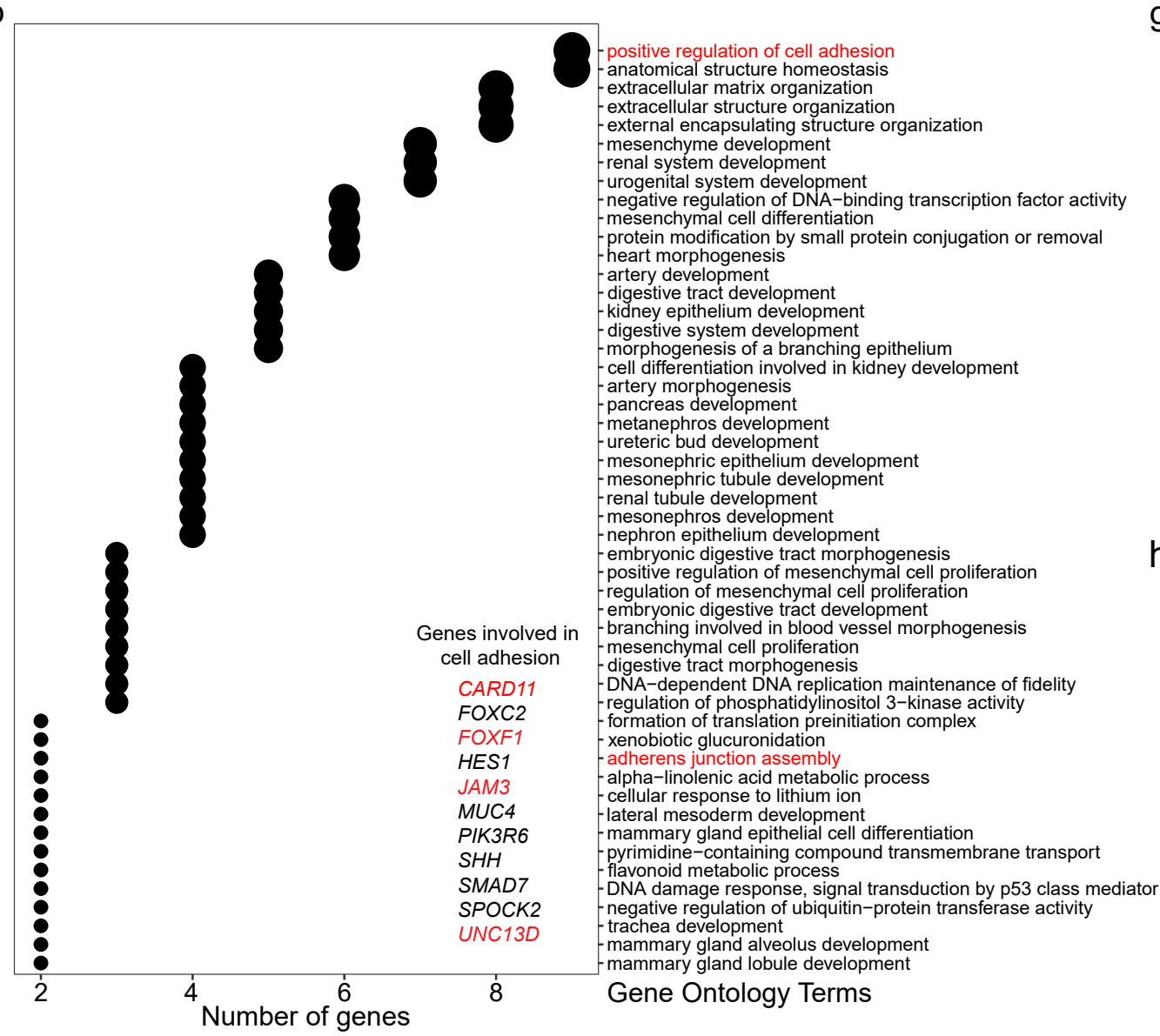

g

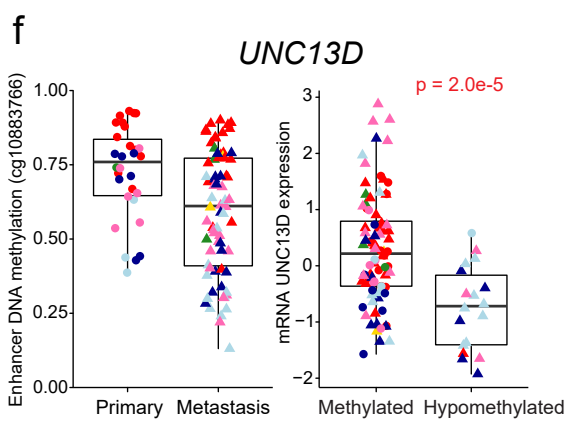

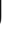

$\square$ Basal $\square$ HER2E $\square$ LumA $\square$ LumB $\square$ Claudin $\square$ Normal $\bigcirc$ Primary $\Delta$ Metastasis

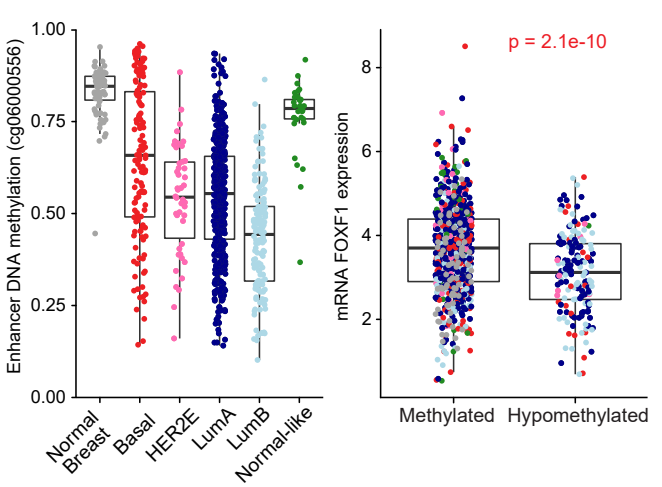

$\mathrm{h}$

TCGA breast cancer

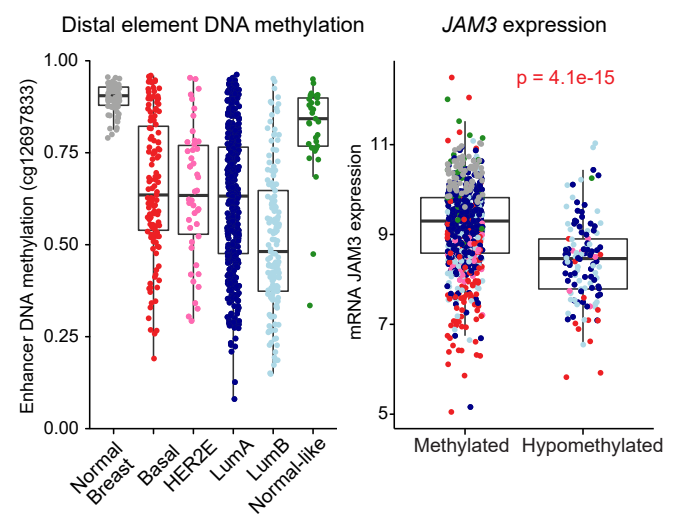


Fig. 4. Metastatic tumor-associated DNA hypomethylation at distal enhancer elements. a, Analysis of DNA binding proteins at the significantly hypomethylated CpG sites. Each dot represents one of the 11,348 ChiP-seq data analyzed. Y-axis represents the odds ratio of enrichment, and $\mathrm{X}$-axis represents the number of significant $\mathrm{CpGs}$ overlapping protein binding sites. The size of the dot denotes the statistical significance of the enrichment (Fisher's exact test). b. Gene Ontology (GO) analysis of putative target genes for the hypomethylated ESRI or FOXA1 distal binding sites. Shown are the top fifty GO terms based on the $\mathrm{P}$ values from Fisher's exact test. Dots sizes are proportional to the number of genes. Red text highlights cell adhesion GO terms and genes of interest. c-f. Analysis of putative enhancer target genes involved in the regulation of cell adhesion. For each gene, a comparison of distal element DNA methylation between primary and metastatic tumors is shown on the left, and putative target gene expression between methylated ( $\beta$-value $\geq 0.4$ ) vs. unmethylated $(\beta$-value of $<0.4$ ) tumors is shown on the right. The $P$ values were calculated using Welch's two-sample $t$-test. $\mathbf{g}, \mathbf{h}$. Analysis of distal element DNA hypomethylation (left) and putative target gene expression (right) in TCGA breast cancer data. Normal breast tissue samples are indicated in dark gray, and tumor samples are color-coded by the PAM50 molecular subtype. The samples were grouped into either methylated or unmethylated using a $\beta$-value threshold of 0.4 . The $P$ values were calculated using Welch's two-sample t-test. 


\section{AER8}
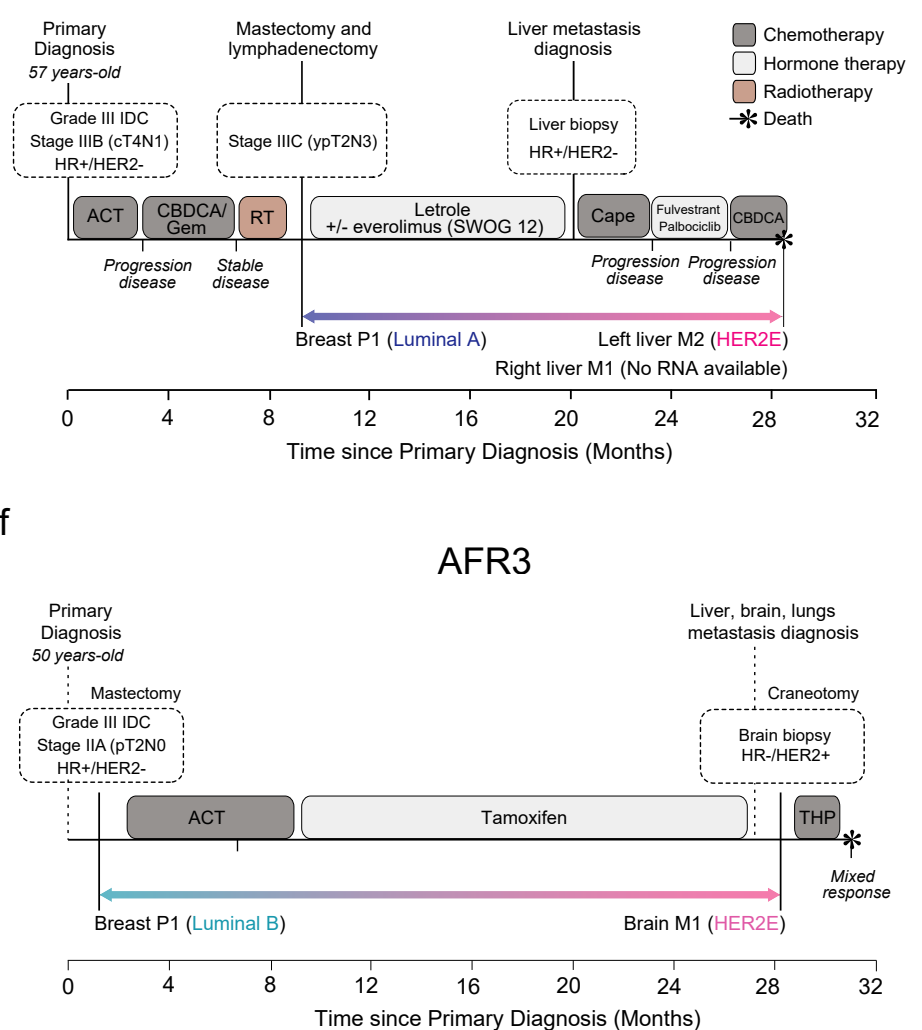

k

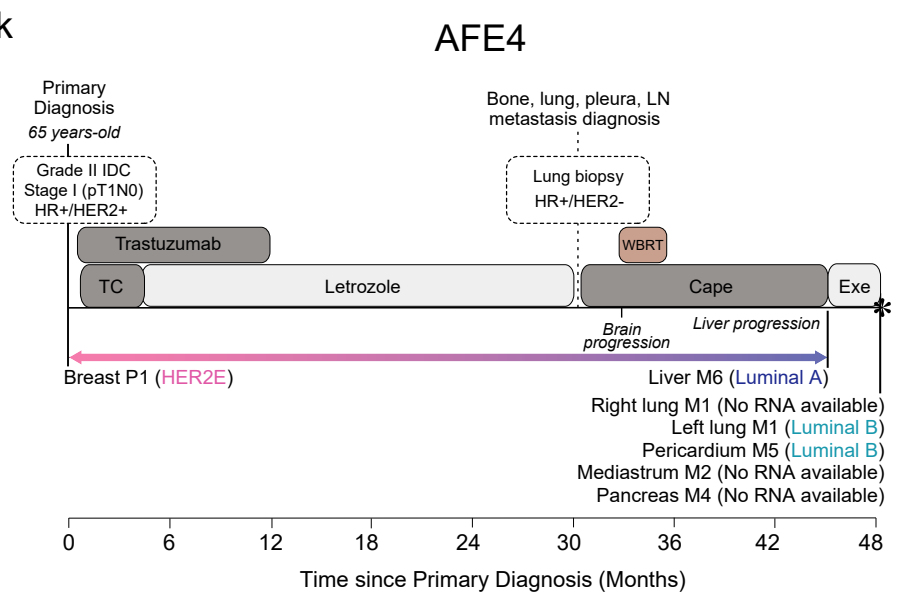

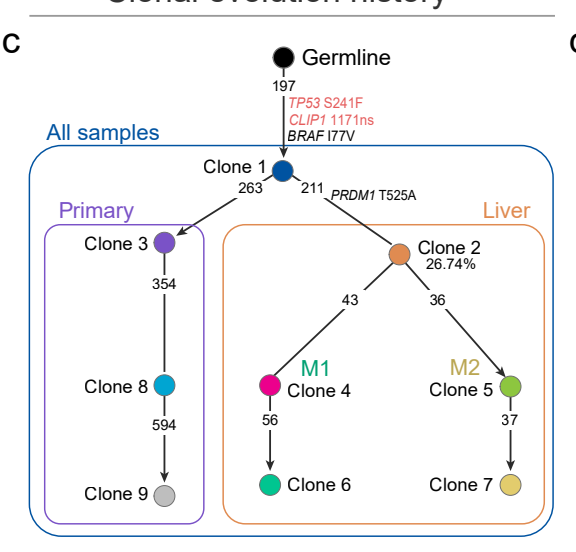

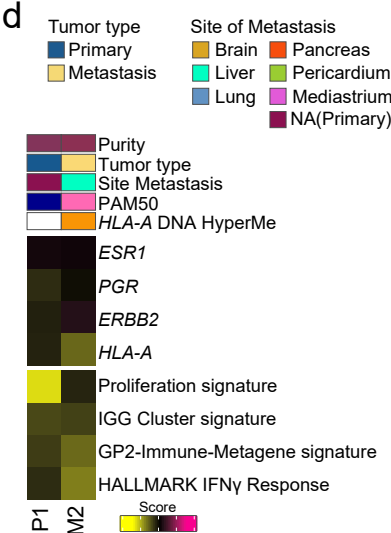

$\mathrm{h}$
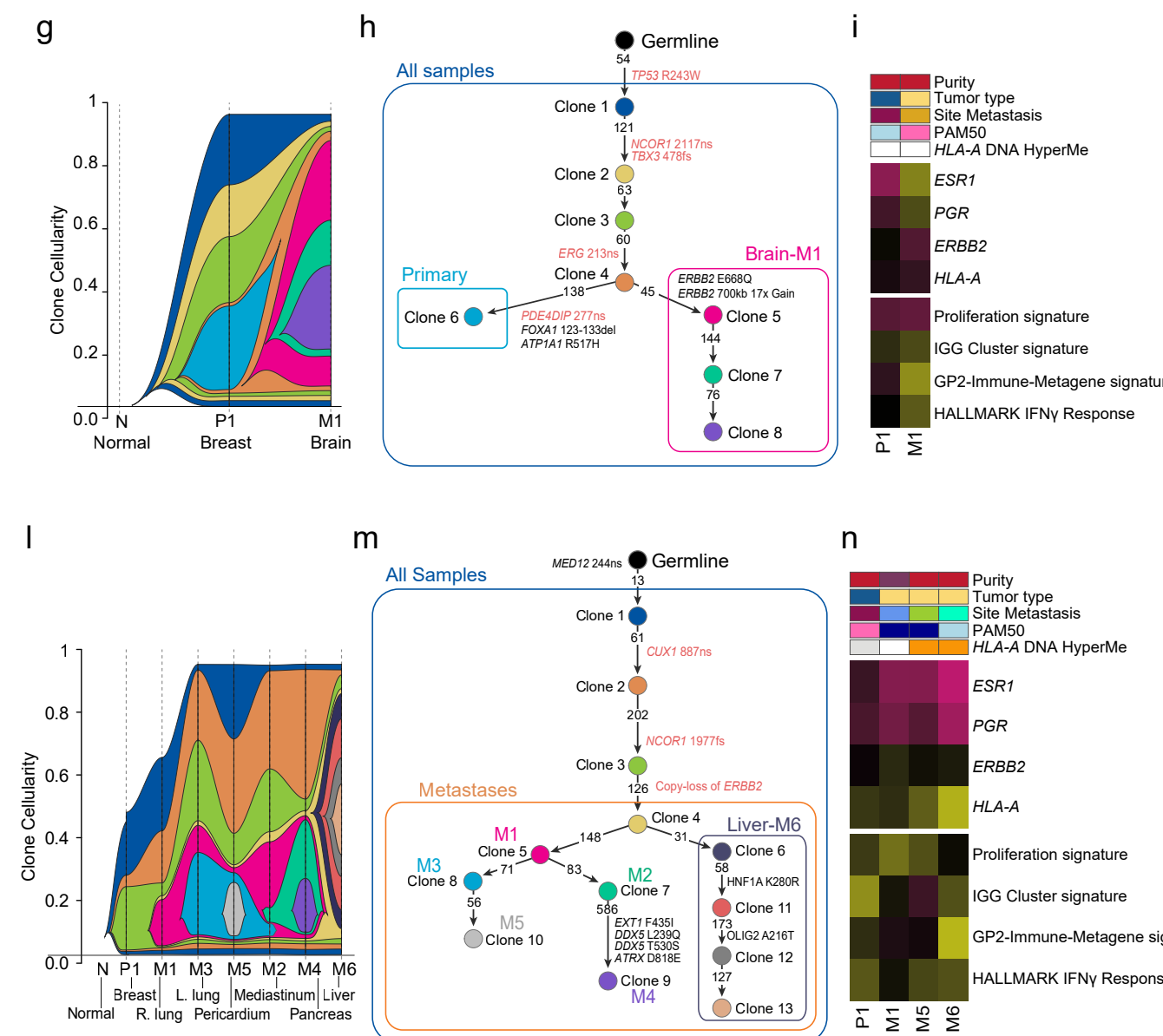

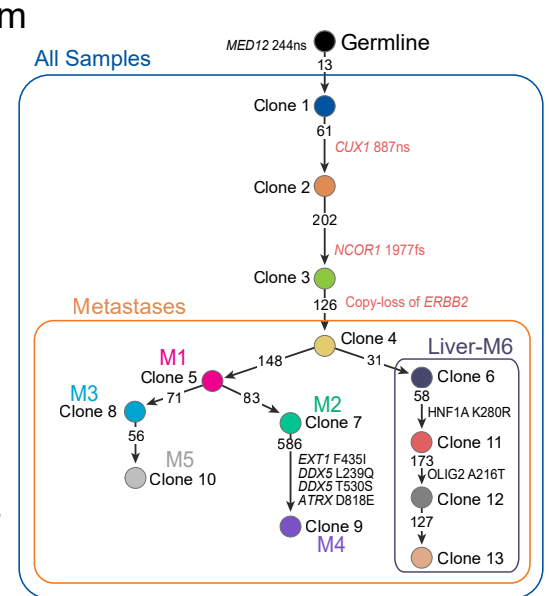

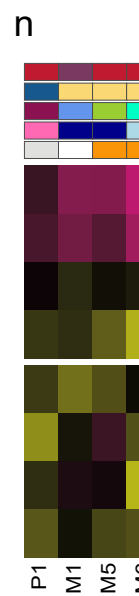

e PAM50 DNA HyperM G HER2E
Luma
Lumb

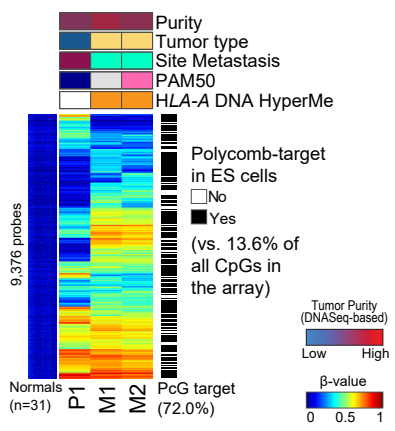

j

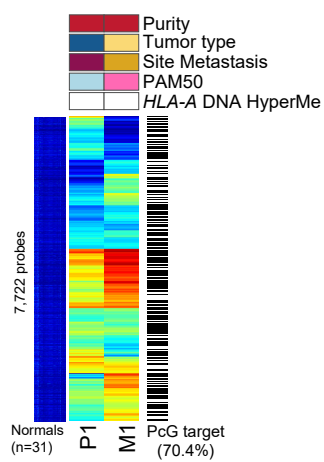

0

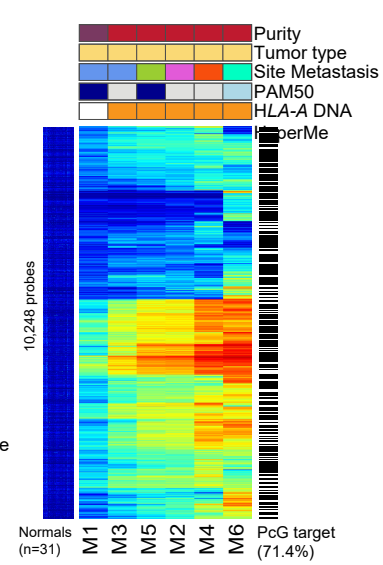


Fig. 5. Multi-omics patient characterization of individual AURORA cases. Timeline of patient clinical history, clonal structure, clonal evolution, transcriptome, and methylome description of patient AER8 (a, b, c, d, e), AFR3 (f, g. g, i, j) and AFE4 (k, l, m, n, o).

Transcriptome data reflects gene expression values and gene expression signatures calculated in using normalized RNAseq data. P, primary; M, metastasis, LumA, luminal A, LumB, luminal B; HyperMe, hypermethylation. 


\section{Supplementary Files}

This is a list of supplementary files associated with this preprint. Click to download.

- GarciaRecio.et.alAURORAMethods.pdf

- SupplementaryTable1.xlsx

- SupplementaryTable2.xIsx

- Supplementarytable3.xlsx

- Supplementarytable4.xlsx

- GarciaRecio.S.et.alAURORAExtendedDataFigures.pdf 